\title{
RDUS
}

Revue de DROIT

UNIVERSITÉ DE SHERBROOKE

Titre : $\quad$ LA GESTION ÉCONOMIQUE DE L'EAU SOUTERRAINE PAR LE DROIT DE PROPRIÉTÉ AU QUÉBEC

Auteur(s): $\quad$ Hugo TREMBLAY

Revue : $\quad$ RDUS, 2007-2008, volume 38, numéro 2

Pages: $\quad 483-539$

ISSN : $\quad 0317-9656$

Éditeur : $\quad$ Université de Sherbrooke. Faculté de droit.

URI : $\quad$ http://hdl.handle.net/11143/11589

DOI : $\quad$ https://doi.org/10.17118/11143/11589 
Page vide laissée intentionnellement. 


\title{
ARTICLE
}

\section{LA GESTION ÉCONOMIQUE DE L'EAU SOUTERRAINE PAR LE DROIT DE PROPRIÉTÉ AU QUÉBEC}

\author{
par Hugo TREMBLAY*
}

Le caractère épuisable de l'eau de même que l'accroissement des usages anthropiques et de la pollution provoquent une raréfaction des ressources hydriques. L'approche économique est fréquemment présentée comme un moyen d'améliorer la gestion de la ressource. Ce texte propose une étude de la relation entre la gestion économique et la propriété de la ressource. L'eau souterraine est plus particulièrement visée compte tenu des liens traditionnels qui l'unissent à la propriété au Québec. Dans un premier temps, ce texte établit que la propriété permet effectivement la gestion économique de l'eau au niveau théorique. Ainsi, dans la mesure où la propriété permet d'appréhender l'eau souterraine, elle permet sa gestion économique. Cependant, une étude attentive du cadre juridique relatif à la propriété démontre que l'eau souterraine n'est jamais l'objet de quelque type de propriété au Québec, qu'elle soit privée, commune ou publique. L'eau souterraine est plutôt une chose commune qui se soustrait au droit de propriété. La gestion économique de l'eau souterraine par le biais de la propriété est donc impossible au Québec.

The constant increase in anthropogenic uses compounded by pollution and modifications in hydrological patterns due to climate change lead to water resources scarcity. In this context, economic management is often portrayed as a means for improved water governance. This article studies the relationship between economic management and property ownership over water resources. Groundwater is the specific focus of discussion given that it is traditionally perceived as an object of private property in Quebec. First, it is established that property can indeed provide a legal framework for economic management of water resources at the theoretical level. Hence, inasmuch as it applies to groundwater, property allows for economic management of the resource in Guebec. However, the study of the legal framework related to property demonstrates that groundwater is never an object of ownership, whether private, public or common. Rather, groundwater is a res communis. As a result, economic management of groundwater through property rights is impossible in Quebec.

* Étudiant au doctorat, UNESCO Centre for Water Law, Policy and Science. 


\section{SOMMAIRE}

\section{LA GESTION ÉCONOMIQUE PAR LA}

1.1. Le cadre hydrogéologique.............................. 489

1.2. Le cadre théorique relatif à la gestion économique par la propriété

1.3. La relation entre la propriété et l'eau souterraine dans le contexte québécois.............. 499

2. LA PROPRIÉTE ET L'EAU SOUTERRAINE................ 506

2.1. La propriété privée et l'eau souterraine .............. 506

2.2. La propriété commune : modalités et démembrements de la propriété privée

2.3. La propriété de l'eau souterraine et l'État .......... 519

2.4. L'eau souterraine : res communis..................... 527 


\section{INTRODUCTION}

L'eau douce est une ressource cruciale. Bien que son renouvellement progressif soit assuré par le cycle hydrologique, la quantité d'eau douce est limitée. L'accroissement constant des usages anthropiques et de la pollution réduit les volumes d'eau disponibles. Dans ce contexte, la gestion des ressources hydriques fait face à d'importants défis ${ }^{1}$. Une piste de solution fréquemment avancée afin d'améliorer la gestion de l'eau repose sur l'approche économique: puisqu'elle est une ressource dont la rareté augmente, l'eau douce peut être considérée comme un bien économique qui requiert une gestion plus efficace ${ }^{2}$. À cet égard, le principe No4 de la Déclaration de Dublin fait souvent office de référence :

Past failure to recognize the economic value of water has led to wasteful and environmentally damaging uses of the resource. Managing water as an economic good is an important way of achieving efficient and equitable use, and

1. Malin Falkenmark, "Water Scarcity - Challenges for the Future» (1997) 21 dans Edward Brans, Esther de Haan, André Nollkaemper \& Jan Rinzema, éd., The Scarcity of Water. Emerging Legal and Policy Responses, International Environmental Law and Policy Series, London, Kluwer Law International, résume ainsi les facteurs contribuant aux défis liés à la gestion de l'eau: "First, the world population is growing at an explosive rate, in particular in the regions where water is scarce for hydroclimatic reasons. [...] Secondly, as quality of life increases along with socio-economic development and successful poverty eradication, demands on water may be expected to increase for household purposes, for industrial activities and generation of employment, and for raising food security in drought-prone low yield agriculture. Thirdly, in spite of at least three decades of efforts to reduce the symptoms of waste mismanagement and the outflow of pollutants to the atmosphere, to landscape and to water bodies, water pollution tends to expand rather than shrink. This reduces the usable amount."

2. Antoinette Hildering, "Water as an Economic Good" (2005) 209 dans Laurence Boisson de Chazournes \& Salman Salman, éd., Les ressources en eau et le droit international, Boston, Martinus Nijhoff Publishers. 
of encouraging conservation and protection of water resources. ${ }^{3}$

Au Québec, la gestion économique de l'eau fait présentement l'objet de réflexions substantielles ${ }^{4}$. Le Ministère du Développement durable, de l'Environnement et des Parcs (ci-après "MDDEP") a produit en 2004 un document d'orientation portant sur l'instauration d'un système de redevances sur l'eau qui vise à appliquer le principe d'utilisateur-payeur et à permettre la récupération, auprès des utilisateurs de l'eau, d'une partie des coûts publics et sociétaux de conservation et de restauration des ressources hydriques ${ }^{5}$. Par ailleurs, les médias ont récemment mentionné la possibilité d'une Bourse de l'eau à Montréal6.

Le droit fait désormais écho à cette tendance en matière de gestion de l'eau. La Loi sur la qualité de l'environnement (ci-après "LGE») prévoit actuellement que le gouvernement peut adopter des règlements pour mettre en place des instruments économiques, notamment des permis négociables ou des redevances liées à l'utilisation et la gestion de l'eau? ${ }^{7}$. L'engagement en faveur d'une

3. Déclaration de Dublin, Conférence internationale sur l'eau et l'environnement, Dublin, janvier 1992, disponible en ligne à l'adresse suivante : http://www.wmo.ch/pages/prog/hwrp/otherdoc.html (consultée le 15 juillet 2008). La Déclaration de Dublin était considérée comme un document préparatoire au Sommet de la Terre de Rio de Janeiro qui eut lieu en 1992. Son objectif était de préciser les modalités d'une saine gestion des ressources hydriques dans le cadre de la mouvance liée au développement durable et issue du Rapport Brundtland.

4. Voir par exemple, La Jeune Chambre de commerce de Montréal, "L'eau : une richesse à conserver et à gérer de façon durable pour le développement du Québec», Montréal, JCCM, 2007 aux pp. 19 et s.

5. Voir Ministère du Développement durable, de l'Environnement et des Parcs, Bilan annuel 2005-2006 de la mise en oeuvre de la Politique nationale de l'eau du gouvernement du Québec, Québec, Gouvernement du Québec, p. 33.

6. Vincent Brousseau-Pouliot, "L'OCDE propose une Bourse de l'eau", La Presse, (11 juin 2008) p. 1 du cahier des affaires.

7. Article 31 al.1 §e.1 LQE, L.R.Q., c. Q-2. Ce pouvoir de réglementer a été introduit dans la LQE par la Loi modifiant la Loi sur la qualité de l'environnement, L.Q., 2001, c. 59. Depuis une modification subséquente 
(2008) 38 R.D.U.S.

La gestion économique

de l'eau souterraine

par le droit de propriété au Québec

gestion économique des ressources hydriques est précisé par le Projet de Loi affirmant le caractère collectif des ressources en eau et visant à renforcer leur protection (ci-après "Projet de loi no92") ${ }^{8}$. Le Projet de loi no92 énonce notamment le principe de l'utilisateurpayeur, qui impliquerait que les coûts liés à l'utilisation des ressources en eau soient assumés par les utilisateurs ${ }^{9}$. De plus, la mise en œuvre du principe de réparation pourrait être liée à une évaluation monétaire de l'altération des propriétés physiques, chimiques ou biologiques, du potentiel écologique, ou de l'état quantitatif des ressources en eau ${ }^{10}$. Finalement, le Projet de loi $n^{\circ} 92$ rendrait cessibles les autorisations de prélèvement de l'eau souterraine alors qu'elles ne le sont pas à l'heure actuelle ${ }^{11}$.

Cependant, l'existence d'un cadre juridique rendant possible la gestion économique de l'eau peut être envisagée sans même tenir compte d'instruments statutaires spécifiques. Le droit de propriété est perçu comme une structure juridique permettant la gestion économique de l'eau depuis longtemps. Si le droit de propriété peut appréhender la ressource hydrique et s'y appliquer, les fondements d'un mécanisme de marché sont établis. Ainsi,

introduite par la Loi modifiant la Loi sur la qualité de l'environnement et autres dispositions législatives, L.Q., 2002, c. 53, les droits ou redevances perçus suite à la mise en œuvre d'un tel règlement doivent être versés dans un fond prévu par la Loi sur le Ministre du Développement Durable, de l'Environnement et des Parcs, L.R.Q., c. M-30.001 (ci-après "LMDDEP»). Selon l'article 15.2 LMDDEP, le Ministre veille à ce que les sommes ainsi versées soient affectées au financement de mesures prises pour assurer la gouvernance de l'eau, entre-autres pour la conserver en qualité et en quantité suffisantes dans une perspective de développement durable.

8. Le récent projet de Règlement sur la déclaration obligatoire des prélèvements d'eau est présenté comme une étape supplémentaire du processus de mise en œuvre d'un cadre de gestion économique des ressources hydriques. Le projet n'est pas encore publié dans la Gazette Officielle mais est disponible en ligne à l'adresse suivante : http:// www.mddep.gouv.qc.ca/eau/consultation/prelevements/regle ment_adm.pdf (consultée le 1er novembre 2008).

9. Loi affirmant le caractère collectif des ressources en eau et visant à renforcer leur protection, P.L. 92, 2008, 1re sess, 38e lég., Québec, art. 4.

10. Projet de loi no 92, art. 6-9.

11. Projet de loi no 92, art. 17-31.84. 
dans la mesure où il est possible d'être propriétaire d'un volume d'eau auquel s'appliquent également d'éventuels instruments statutaires de gestion économique, deux cadres juridiques visant le même objet pourraient se chevaucher ${ }^{12}$. Une telle situation pourrait notamment entraver l'articulation cohérente et l'application optimale des mécanismes statutaires d'allocation des eaux souterraines.

Cette étude a pour objectif d'explorer la relation entre la gestion économique de l'eau et la propriété de la ressource au Québec. À cette fin, l'eau souterraine est utilisée comme exemple puisqu'elle est fréquemment présentée comme un objet de propriété, contrairement à l'eau de surface ${ }^{13}$. Les nombreux instruments statutaires qui peuvent exercer une influence importante sur l'exercice du droit de propriété à l'égard de la ressource sont exclus du champ de l'étude afin de cibler spécifiquement l'interaction entre la propriété et la gestion économique ${ }^{14}$. Pour déterminer si le droit de propriété permet effectivement la gestion économique de l'eau souterraine à l'état naturel au Québec, il est d'abord nécessaire d'analyser comment la propriété de la ressource est liée à la gestion économique (1.), et ensuite comment le droit de propriété appréhende l'eau souterraine (2.).

12. À cet égard, l'article 1 du Projet de loi no92 énonce que l'eau de surface et l'eau souterraine, dans leur état naturel, sont des ressources qui ne peuvent être appropriées sauf lorsque la loi, et notamment le Code civil, le permet.

13. Aux fins de la présente étude, la définition de l'eau souterraine exclut les eaux se trouvant sous terre dans des citernes, conduits et autres contenants créés par l'être humain.

14. Sont notamment exclus du champ d'étude le Règlement sur le captage des eaux souterraines, D.696-2002, 12 juin 2002, G.O.Q. II No24A, p. 3539 [R.Q., c. Q-2, r.1.3], et la Loi sur la protection du territoire et des activités agricoles, L.R.Q. c. P-41.1 (ci-après "LPTAA"). Ces deux instruments juridiques peuvent subordonner l'utilisation de l'eau souterraine à des autorisations dans leurs champs d'application respectifs. 


\section{LA GESTION ÉCONOMIgUE PAR LA PROPRIÉTÉ}

Pour déterminer comment la propriété est liée à la gestion économique de la ressource, il est d'abord nécessaire de comprendre le fonctionnement des systèmes hydrogéologiques. Cette partie commence donc par un bref survol des caractéristiques physiques de l'eau souterraine qui ont un impact sur sa gestion (1.1.). Ensuite, la relation entre la gestion économique de la ressource et le droit de propriété est établie au niveau théorique (1.2.). Finalement, les paramètres spécifiques au cadre juridique québécois qui permettent l'incarnation du cadre théorique sont identifiés (1.3.).

\subsection{Le cadre hydrogéologique}

Les caractéristiques physiques de la ressource imposent des contraintes fondamentales qui ont un impact important sur certains aspects de la gestion des eaux souterraines. En premier lieu, il importe de présenter un portrait sommaire de l'hydrogéologie puis d'identifier certaines caractéristiques de la ressource qui se répercutent sur sa gestion.

L'eau souterraine ne court pas en rivières le long de tunnels souterrains pour se jeter dans de vastes cavernes où se forment des lacs. Au Québec, l'eau souterraine est omniprésente dans le sol à faible profondeur. L'eau souterraine peut être définie simplement comme l'eau qui se trouve sous la surface du sol dans les pores, les interstices et les fractures des diverses formations géologiques ${ }^{15}$. L'eau souterraine s'infiltre dans le sol parce qu'il n'est pas parfaitement compact; les espaces existants dans les matières granulaires ou fracturées qui composent le sol sont occupés par l'eau de la même façon que l'eau imbibe une éponge. Une nappe d'eau souterraine se forme lorsque l'eau sature les espaces interstitiels de la matière solide qui compose le sol ${ }^{16}$. Une

15. Vishwambhar Prasad Sati, Water resource management and perspectives, Jaipur, Pointer Publishers, 2004, à la p. 175.

16. Les formations géologiques qui contiennent de l'air, c'est-à-dire qui ne sont pas saturées d'eau mais seulement humides, se trouvent en surface 
nappe phréatique est une nappe d'eau souterraine suffisamment importante pour être captée de façon utile par un puits ${ }^{17}$.

Généralement, l'eau s'infiltre dans le sol à partir de la surface par l'effet de la gravité. Toutefois, sous l'influence de l'agencement et de la composition des formations géologiques, l'eau souterraine s'écoule principalement en direction horizontale. Toutes les formations géologiques qui composent le sol à moindre profondeur ne sont pas également perméables. Leur perméabilité dépend du type de matériel qui les compose ${ }^{18}$. Certaines d'entre elles sont pratiquement imperméables. Les formations géologiques imperméables sont désignées sous le terme d'aquitard, tandis que les formations géologiques perméables sont des aquifères. La disposition des aquifères et des aquitards dans le sol dicte l'écoulement de l'eau souterraine, que la gravité mène de l'amont d'une pente à l'aval : l'eau coule vers l'aval en suivant la pente de l'aquitard qui forme la limite inférieure de l'aquifère contenant la nappe souterraine ${ }^{19}$.

du sol et forment une couche d'épaisseur variable appelée zone vadose. L'eau contenue dans la zone vadose ne peut être captée utilement par un puits; seule l'eau souterraine d'une zone saturée peut l'être. Les nappes d'eau souterraine se trouvent sous la surface de saturation.

17. Henrik Rasmussen, Alain Rouleau et Sylvie Chevalier, dir., Outils de détermination d'aires d'alimentation et de protection de captage d'eau

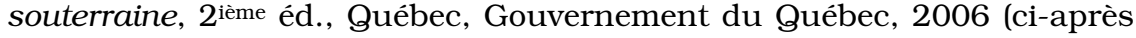
"Outils de détermination"), à la p. 2-7.

18. Le degré de perméabilité des aquifères détermine la vitesse de circulation de l'eau dans le sol : Raynald Lacouline, dir., Guide: les périmètres de protection autour des ouvrages de captage de l'eau souterraine, Québec, Publications du Québec, 1995, à la p. 40. Par exemple, la vitesse de circulation de l'eau souterraine varie de plusieurs mètres par jour à quelques centimètres par siècle: Environnement Canada, Les eaux souterraines, trésors cachés de la nature, Ottawa, Gouvernement du Canada, 1993, à la p. 2.

19. La nappe d'eau souterraine dans un aquifère qui n'est pas recouvert d'un aquitard est une nappe libre. Lorsqu'un aquifère contenant une nappe d'eau souterraine est recouvert d'un aquitard, la nappe d'eau est dite captive ou semi-captive. Un tel aquifère est donc contenu entre deux aquitards qui forment son "plancher" et son "plafond". L'aquitard qui forme le "plafond" de l'aquifère empêche que la nappe captive soit rechargée par l'eau de surface dans la zone où il chevauche l'aquifère. 
(2008) 38 R.D.U.S.

La gestion économique

de l'eau souterraine

par le droit de propriété au Québec

Les captages anthropiques exercent aussi une influence sur l'écoulement de l'eau souterraine. Autour d'un ouvrage de captage, il existe une aire d'alimentation dans laquelle toute l'eau souterraine s'écoule vers l'ouvrage pour y être captée. Le pompage de l'eau par un puits génère dans son voisinage une zone de dépression de la surface de la nappe phréatique en forme de cône : il s'agit du rabattement, qui augmente plus la distance par rapport à l'ouvrage de captage est petite ${ }^{20}$.

Par ailleurs, en matière de gestion des eaux souterraines, deux contraintes fondamentales s'imposent. Premièrement, le sol contient une quantité finie d'eau souterraine en un moment donné. L'eau souterraine fait partie intégrante du cycle hydrologique, qui constitue un circuit fermé transférant en boucle un volume d'eau limité21. Bien que l'eau souterraine soit assez

Selon Gabriel Eckstein \& Yoram Eckstein, "A Hydrological Approach to Transboundary Ground Water Resources and International Law", (2003) Vol.19, Am. U. Int'l L. Rev., 201, à la p. 212, et Marcella Nanni \& Stephen Foster, "Groundwater resources : shaping legislation in harmony with real issues and sound concepts", (2005) Vol.7, Water Policy, 543 (ci-après "Shaping legislation"), à la p. 545, les nappes captives ne sont pas coupées de tout lien avec l'ensemble du cycle hydrologique mais en font partie intégrale. Elles sont rechargées par l'écoulement latéral de l'eau souterraine à partir de fenêtres de recharge où l'aquifère n'est pas recouvert par un aquitard, possiblement dans des zones très éloignées en amont hydraulique. Les nappes captives peuvent aussi se décharger dans les eaux de surface en aval.

20. Ce commentaire vise en particulier les ouvrages qui captent une nappe phréatique libre. Néanmoins, il est possible d'avancer, à l'instar de Stephen Foster, "Essential Concepts for Groundwater Regulators" dans Salman Salman, dir., Groundwater: legal and policy perspectives, Washington, World Bank, 1999, à la p. 21 (ci-après "Essential concepts"), que tout captage d'eau souterraine résulte en un rabattement d'une nappe d'eau souterraine sur une certaine surface.

21. Global Water Partnership Technical Advisory Committee, Integrated Water Resources Management, Stockholm, Global Water Partnership, 2000 (ci-après "GWP IWRM"), à la p. 14. L'eau souterraine provient de l'eau de pluie qui tombe au sol et s'y infiltre immédiatement, ou qui ruisselle à sa surface dans les cours d'eau et les lacs, ou s'accumule sous forme de neige et de glace avant de pénétrer le sol. Globalement, les masses terrestres reçoivent $36 \times 10^{12} \mathrm{~m}^{3}$ sur une base annuelle d'eau provenant des océans. Un volume équivalent s'écoule des masses 
abondante au Québec et que le stock disponible de la ressource se renouvelle, il en existe une quantité limitée ${ }^{22}$. Le volume maximal d'eau souterraine disponible en un moment précis aux niveaux local, régional et provincial constitue donc une contrainte fondamentale en matière de gestion de la ressource. La quantité d'eau souterraine disponible impose une limite inéluctable à l'augmentation des usages anthropiques des eaux souterraines. Une augmentation des usages anthropiques qui ne tient pas compte de cette limite entraîne une surexploitation de la ressource et provoque un stress hydrique ${ }^{23}$.

Deuxièmement, la connaissance du système hydrogéologique est nécessairement approximative et imparfaite. Le système hydrologique est extrêmement complexe et une modélisation adéquate de la multitude de facteurs exerçant une influence sur l'eau souterraine dépasse les capacités techniques actuelles. L'eau souterraine provient de l'ensemble des sources de surface qui constitue la recharge. Les eaux souterraines s'écoulent dans les formations géologiques sur des distances variables, puis elles retournent à la surface sous l'action de la végétation, de l'être humain ou de l'écoulement naturel dicté par le milieu géologique. L'hétérogénéité géologique et les variations météorologiques ont un impact déterminant à cet égard, ce qui implique une marge d'incertitude significative restreignant la validité des modèles et

terrestres vers les océans à chaque année: François Anctil, Jean Rousselle \& Nicolas Lauzon, Hydrologie, cheminements de l'eau, Montréal, Presses internationales Polytechnique, 2005, aux pp. 2-5.

22. Traditionnellement, les réserves d'eau souterraine au Québec sont estimées à $200000000000 \mathrm{~m}^{3}$ d'eau : Marcel Sylvestre \& Claude Grenier, L'eau souterraine, une ressource à exploiter, Sainte-Foy, Gouvernement du Guébec, 1987, à la p. 11. Depuis, cette estimation est généralement reprise.

23. Selon Ministère de l'Environnement et de la Faune du Québec, La problématique des eaux souterraines au Québec, Projet, Québec, Gouvernement du Québec, 1996 (ci-après "La problématique des eaux souterraines"), à la p. 16, la surexploitation peut causer l'abaissement graduel des niveaux d'eau souterraine, la diminution des débits des cours d'eau, des sources et des débits exploités par les ouvrages de captage, la réduction des zones marécageuses, la dégradation de la qualité de l'eau et l'affaissement des sols. 
(2008) 38 R.D.U.S.

La gestion économique

de l'eau souterraine

493

par le droit de propriété au Québec

des études scientifiques en matière d'hydrogéologie ${ }^{24}$. De plus, en faisant résurgence dans les eaux de surface, l'eau souterraine permet le maintien du régime hydrique et assure la santé des écosystèmes ${ }^{25}$. La modification d'un paramètre dans le cycle hydrologique entraîne donc des conséquences innombrables dont il est impossible de prévoir avec certitude les impacts sur les multiples usages anthropiques et environnementaux de l'eau. En somme, la portée et l'efficacité des outils scientifiques relatifs aux eaux souterraines restent limitées compte tenu de l'ampleur et de la complexité du système hydrologique et des écosystèmes qui en dépendent.

\subsection{Le cadre théorique relatif à la gestion économique par la propriété}

Il existe plusieurs façons de concevoir la gestion économique de l'eau. Toutes les approches économiques de la gestion de la ressource ne sont pas liées au droit de propriété. Ainsi en est-il de la tarification de l'approvisionnement en eau potable par aqueduc, qui n'implique pas que les usagers du service disposent d'un droit de propriété sur l'eau qu'ils consomment. En fait, la tarification des quantités d'eau utilisées à différentes fins reflète souvent une approche économique de nature comptable qui vise la récupération des coûts engendrés par l'usage de la ressource. Les approches économiques de la gestion de la ressource indépendantes du droit de propriété ne font pas l'objet de cette étude, qui a pour objet exclusif le lien entre la propriété de la ressource et la gestion économique.

La théorie micro-économique orthodoxe veut que l'assignation d'une valeur monétaire à l'eau permette de parvenir à

24. Alfonso Rivera, "How Well Do We Understand Groundwater in Canada? A Science Case Study" dans Linda Nowlan, Buried Treasure. Groundwater Permitting and Pricing in Canada, Toronto, Walter \& Duncan Gordon Foundation, 2005, 4 à la p. 7 (ci-après "A Science Case Study").

25. Les interactions entre les eaux de surface et les eaux souterraines sont importantes au point que les deux types de ressource sont souvent indissociables : Outils de détermination, supra note 17, à la p. 2-2 et p. 67. 
un prix qui égalise les coûts et les bénéfices marginaux pour ses utilisateurs par l'opération des mécanismes de marché afin d'atteindre l'efficacité économique et de maximiser la somme des bénéfices tirés de l'usage de la ressource ${ }^{26}$. Selon ce raisonnement, si la valeur économique d'un volume d'eau est supérieure lorsque la ressource est utilisée à une fin particulière plutôt qu'à une autre, le marché se charge d'allouer l'eau à cette fin pour maximiser la richesse globale tirée de l'utilisation de la ressource ${ }^{27}$. Par exemple, si les bénéfices marginaux tirés d'un volume d'eau additionnel sont de $10 \$$ lorsque la ressource est utilisée à des fins industrielles mais seulement de $9 \$$ lorsqu'utilisée à des fins agricoles, une gestion économique efficace requiert l'allocation des volumes d'eau à des fins industrielles plutôt qu'agricoles tant qu'il existe une différence de valeur entre les bénéfices marginaux respectifs ${ }^{28}$. Autrement dit, la gestion de l'eau en tant que bien économique est alors un moyen d'allocation qui vise à répartir la ressource de façon efficace entre différents usages anthropiques en fonction de son utilité - c'est-àdire de sa valeur économique - auprès de ceux-ci.

Selon cette hypothèse, il semble que l'efficacité économique soit maximisée par l'opération d'une structure institutionnelle de marché : "no institutional form can do a better job of advancing

26. Voir notamment Frank Ward, "Decision support for water policy : a review of economic concepts and tools", (2007) Vol. 9, Water Policy, 1; Ronald Griffin, Water resource economics: the analysis of scarcity, policies and projects, Cambridge, MIT Press, 2006 (ci-après "Water resource economics"), aux pp. 11-56; Nicolas Spulber \& Asghar Sabbaghi, Economics of Water Ressources : From Regulation to Privatization, 2 ième éd., Norwell, Kluwer Academic Publishers, 1998, aux pp. 1-72; David Batten, "Can economists value water's multiple benefits?", (2007) Vol.9, Water Policy, 345.

27. Selon Terry Anderson \& Pamela Snyder, Water Markets: Priming the Invisible Pump, Washington, Cato Institute, 1997, à la p. 18: "In the traditional approach, good resource allocation is supposed to come from a careful weighting of the additional (marginal) benefits of employing a resource in one use relative to the additional (marginal) cost of not using it elsewhere."

28. Ibid. 
(2008) 38 R.D.U.S.

La gestion économique

de l'eau souterraine

495

par le droit de propriété au Québec

economic efficiency than markets» 29 . Or, l'existence d'un marché dépendrait de l'existence d'une structure juridique spécifique : la propriété privée 30 . Selon la théorie libérale, "[p]rivate property rights are the basic building blocks of economic freedom»31. Comme pour l'ensemble des autres biens soumis à l'économie de marché, le fonctionnement d'un marché visant la gestion économique de l'eau dépendrait aussi de l'existence de droits de propriété sur la ressource, c'est-à-dire de la reconnaissance juridique de l'appropriation de l'eau par ses utilisateurs :

To the extent that water rights are allowed to become real and personal property and to the extent that they are transferable, it would be possible to rely on the market and individual decision-making to allocate water resources to 'their highest use'. The argument for treating water rights in this fashion are the same as those justifying the market process and individual decision-making in the use of all our resources, i.e., when they provide for maximum production and efficiency consistent with individual freedom of choice. ${ }^{32}$

Ainsi, la reconnaissance de l'eau en tant que bien économique et en particulier l'opération du marché pour son allocation sont étroitement liées au cadre juridique de gestion de la ressource, et plus particulièrement au droit de propriété. Par ailleurs, il est généralement avancé que les droits de propriété applicables à l'eau doivent cumuler les principales caractéristiques suivantes ${ }^{33}$ : définir et établir précisément la

29. Water resource economics, supra note 26, à la p. 203.

30. Voir Friedrich von Hayek, The Constitution of Liberty, Londre, Routledge, 2006, aux pp. 122-124, et John Chapman, "Justice, Freedom and Property" dans Roland Pennock \& John Chapman, Property. Nomos XXII, New York, New York University Press, 1980, 289, p. 313.

31. Walter Block, "Private Property Rights, Economic Freedom and Professor Coase : A Critique of Friedman, McCloskey Medema, and Zorn", (2003) Vol.26 No3, Harv. J. of L. \& Public Pol., 923, à la p. 923.

32. J.W. Milliman, "Water Law and Private Decision Making: A Critique", (1959) Vol.2, J. of L. \& Econ., 41, à la p. 45.

33. Voir notamment William Easter, Mark Rosegrant \& Ariel Dinar, dir., Markets for water: potential and performance, New York, Kluwer Academic 
quantité et la qualité d'eau sujette au droit de propriété; rompre les liens juridiques entre les droits de propriété de l'eau et les droits de propriété du sol de façon à ce que les uns soient indépendants des autres; permettre le transfert des droits de propriété sur l'eau d'une personne à l'autre contre compensation économique; être permanents ou valides sur une période suffisamment longue pour avoir une réelle valeur par rapport à des acheteurs potentiels; être protégés par l'Administration et les tribunaux, ce qui implique que les droits de propriété sur l'eau soient opposables aux tiers et, le cas échéant, justiciables.

D'emblée, cette présentation sommaire des principes relatifs à la gestion de l'eau en tant que bien économique par l'intermédiaire des mécanismes du marché et de la propriété suscite un constat: au Québec, la structure afférente au droit commun de la propriété prévu par le Code civil du Québec (ciaprès "CCQ") peut fournir un cadre juridique qui rende possible la gestion économique de l'eau en lui allouant une valeur monétaire basée sur son coût marginal ${ }^{34}$. Le droit de propriété prévu par le CCQ correspond d'ailleurs à la définition libérale maximaliste de la

Publishers, 1998; Mateen Thobani, "Formal Water Markets: Why, When, and How to Introduce Tradable Water Rights", (1997) Vol.12 No2, World Bank Research Observer, 161; William Easter, Mark Rosegrant \&Ariel Dinar, "Formal and Informal Markets for Water: Institutions, Performance and Constraints", (1999) Vol.14 №1, World Bank Research Observer, 99 (ci-après "Formal and Informal Water Markets"); Carter Ruml, "The Coase Theorem and Western U.S. Appropriative Water Rights", (2005) Vol.45, Nat. Resources J., 169; Miguel Solanes, "Institutional and Legal Issues Relevant to the Implementation of Water Markets", 69, dans Salman Salman, dir., Groundwater. Legal and Policy Perspectives, Washington, World Bank, 1999; Larry Simpson, "Conditions for successful water marketing", 97, dans Guy Le Moigne, William Easter, Walter Ochs \& Sandra Giltner, Water Policy and Water Markets, Washington, World Bank, 1992; Water resource economics, supra note 26, aux pp. 203-242.

34. Voir les articles 947 et 952 C.c.Q., L.Q., 1991, c. 64. Pour les caractéristiques et attributs de la propriété en droit québécois, voir Denys-Claude Lamontagne, Biens et propriété, 5ième éd., Cowansville, Éditions Yvon Blais, 2005 (ci-après "Biens et propriété»), aux pp. 153-157. 
propriété35. Ce constat est confirmé par l'observation : le droit commun de la propriété fournit le cadre légal nécessaire à l'opération des mécanismes de l'offre et de la demande pour l'eau embouteillée, qui est soumise à un prix en fonction du marché à partir de la transaction qui suit son embouteillage. Les trois composantes essentielles de ce droit de propriété sont: 1) la sécurité du titre qui est assurée par la permanence et l'exclusivité du droit de propriété de même que par l'opération des pouvoirs judiciaires et exécutifs de l'État; 2) sous réserve des exigences réglementaires applicables, l'importante autonomie du titre sur un volume clairement défini d'eau, et notamment son indépendance de tout droit de propriété immobilière; 3) la capacité de disposer du bien librement afin de le faire fructifier ou d'en tirer profit, qui se traduit par l'usus, le fructus et l'abusus. A priori, le droit commun de la propriété peut donc satisfaire les caractéristiques requises pour l'opération d'un marché économique de l'eau lorsqu'il s'applique à la ressource.

L'analyse de la gestion économique de l'eau souterraine par la propriété que propose la présente étude est effectuée dans ce contexte. Dès lors, il s'agit d'établir que l'eau souterraine est soumise au droit de la propriété au Québec pour conclure que sa gestion économique est ainsi rendue possible, abstraction faite du droit statutaire. Cependant, une dernière ambiguïté doit être écartée avant de poursuivre la réflexion. Le chevauchement des domaines économique et juridique engendre des glissements sémantiques. La littérature économique identifie généralement comme droit de propriété tout droit d'usage de l'eau correspondant aux caractéristiques qui sont détaillées plus haut, quelque soit la qualification juridique de ce droit. Ainsi, un droit d'usage qui permet l'utilisation de l'eau souterraine grâce à l'obtention d'une licence et qui résulte de la mise en ouvre d'instruments statutaires peut être considéré, d'un point de vue économique, comme un droit de propriété sur l'eau s'il répond aux critères requis. Toutefois, cette étude emprunte principalement une

35. Voir Tony Honoré, Making Law Bind, Essays Legal and Philosophical, Oxford, Clarendon Press, 1987, aux pp. 165-179. 
approche juridique; elle se restreint à l'analyse de la relation entre la gestion économique et les artefacts juridiques qui peuvent effectivement être catégorisés comme droit de propriété. Pour les fins de l'étude, l'ensemble des formes juridiques de la propriété dont l'impact en matière de gestion économique est analysé se restreint aux archétypes suivants :

- le libre accès, c'est-à-dire l'absence de droits de propriété clairement définis sur la ressource. Ceci est largement le cas pour les océans ou l'atmosphère;

- la propriété privée permet d'exclure les non-titulaires de l'usage de la ressource grâce, le plus souvent, à la puissance publique qui en reconnaît et garantit la légitimité. Les droits appartiennent soit à une personne privée, soit à une personne morale. En outre, la propriété privée confère des droits exclusifs et transférables à autrui ce qui la différencie radicalement du libre accès;

- la propriété commune est possédée par une communauté clairement définie d'utilisateurs interdépendants qui à la fois excluent les non-titulaires et définissent les règles d'utilisation. À l'intérieur de la communauté, les droits ne sont ni exclusifs, ni transférables. Le plus souvent, il s'agit d'un accès égal à la ressource[...] Les droits du groupe peuvent faire l'objet d'une reconnaissance officielle par les pouvoirs publics ou simplement de facto;

- la propriété publique implique que la puissance publique détient l'ensemble des droits sur la ressource et définit les modalités d'accès et d'exploitation. En outre, à la différence de la propriété privée, les pouvoirs publics détiennent le pouvoir de police. Enfin, la propriété publique ne fait généralement pas l'objet de transaction sur un libre marché. 36

\section{Cette typologie a l'avantage de présenter les différentes formes de propriété potentiellement applicables aux eaux}

36. Max Falque, "Des droits de propriété sur l'eau, pourquoi pas?" dans Max Falque \& Michel Massenet, dir., Droits de propriété, économie et environnement. Les ressources en eau, Paris, Dalloz, 2000, 1 à p. 6. 
(2008) 38 R.D.U.S.

La gestion économique

de l'eau souterraine

par le droit de propriété au Québec

souterraines de façon limpide, et de délimiter les contours de l'étude juridique de la gestion économique de la ressource par la propriété. Cependant, cette typologie repose sur des simplifications qui font abstraction de certaines possibilités et des formes particulières du droit québécois. La prochaine sous-partie a pour objet de présenter les paramètres spécifiques au cadre juridique québécois qui constituent le contexte de l'étude.

\subsection{La relation entre la propriété et l'eau souterraine dans le contexte québécois}

Au Québec, le droit commun de la propriété est prévu par le CCQ. L'étude de la relation entre la propriété et l'eau souterraine est fortement tributaire du droit civil.

L'eau souterraine est un objet matériel concret. Pour offrir une structure juridique permettant la gestion de l'eau souterraine, le droit civil doit qualifier la ressource comme un bien ${ }^{37}$. Or, un bien est une chose qui est appropriée ou susceptible de l'être ${ }^{38}$. Par ailleurs, le droit civil québécois ne peut appréhender la relation entre la personne et la ressource que par l'intermédiaire des droits réels ${ }^{39}$. Tous les droits réels sont conçus à partir de la

37. Voir Sylvio Normand, Introduction au droit des biens, Montréal, Wilson \& Lafleur, 2000 (ci-après "Introduction au droit des biens"), à la p. 30. Seul le livre troisième du C.c.Q., "Des biens dans leur rapports avec ceux qui y ont des droits et qui les possèdent", offre au droit civil une façon de traiter la ressource. L'article 911 C.c.Q. en présente un panorama complet. La lecture de cet article et la structure du C.c.Q. confirment que le droit civil ne peut fournir un traitement juridique détaillé de l'eau souterraine que si elle est un bien. D’ailleurs, le Code Napoléon, dont est issu le C.c.Q, ne se préoccupe que des res singulorum, soit les objets susceptibles de propriété privée : Antoine Frenet, Recueil complet des travaux préparatoires du Code civil, Osnabrück, Otto Zeller Verlag, 1968, Tome 11, aux pp. 39-40.

38. Gil Rémillard, Code civil du Québec: commentaires du ministre de la Justice et Loi sur l'application de la réforme du Code civil du Québec, Montréal, Publications DAFCO, 1993 (ci-après "Commentaires du ministre»), à p. 306.

39. Simoneau c. Berthiaume, [1998] R.D.I. 373 (C.A.) (ci-après "Simoneau c. Berthiaume"), à la p. 376. Notons qu'il n'est pas possible d'écarter a priori 
propriété privée 40 . Pour y parvenir, il suffit soit de décomposer la propriété et d'en modifier le contenu, soit de ne retenir que certains aspects de son régime juridique ${ }^{41}$. Les droits réels procèdent de la propriété parce qu'elle contient en germe l'ensemble des services et utilités susceptibles d'être tirés d'un bien $^{42}$.

Si elle est possible, la propriété de l'eau souterraine permet le contrôle le plus complet de la ressource. En particulier, le droit de propriété est un mode de relation par rapport à un bien qui emporte le droit d'en user, d'en tirer des revenus, et d'en disposer de façon exclusive, perpétuelle, absolue ${ }^{43}$. Il consacre la liberté d'agir du propriétaire, dont la volonté constitue l'élément moteur essentiel du droit des biens ${ }^{44}$. Or, la volonté individuelle s'exprime essentiellement par le biais de vecteurs économiques ${ }^{45}$.

l'étude de certains pouvoirs exercés en marge des droits réels, tels l'administration du bien d'autrui ou la fiducie. En effet, de tels pouvoirs peuvent se révéler pertinents, notamment parce que l'État se présente comme fiduciaire de l'eau : à ce sujet, voir ci-dessous 2.3.

40. Introduction au droit des biens, supra note 37 , à la p. 77 , exprime ainsi le caractère central de la propriété en droit civil : "Le titre de la propriété est l'un des plus importants du Code, étant donné les liens étroits qui l'unissent avec les autres parties du Code. Ce titre établit ce qu'est la propriété alors que le livre sur les personnes mentionne qui peut acquérir la propriété. Les livres consacrés aux successions, aux obligations, aux priorités et aux hypothèques, et à la prescription traitent des moyen d'acquérir la propriété."

41. Christian Atias, Droit civil. Les biens, 5ième éd., Paris, Litec, 2000, aux pp. 57-58, 66.

42. François Frenette, "Bilan de la réforme du droit des biens", (2003) Vol.105, R. du N., 309 (ci-après "Bilan de la réforme»), p. 317; Introduction au droit des biens, supra note 37, à la p. 77 .

43. Art. 947 C.c.Q; Biens et propriété, supra note 34, aux pp. 154-157. Toutefois, chacune des caractéristiques du droit de propriété peut faire l'objet de limites ou restrictions.

44. Introduction au droit des biens, supra note 37, aux pp. 8-9. Dans une perspective environnementale, voir Agathe Van Lang, Droit de l'environnement, Paris, Presses universitaires de France, 2002 (ci-après "Droit de l'environnement»), aux pp. 146-148.

45. Selon Jean Carbonnier, Droit civil. Les biens, 18ième éd., Tome 3, Paris, Presses universitaires de France, 1997 (ci-après "Droit civil. Les biens"), à la p. 15 : "Tout dans notre civilisation juridique, s'exprime en monnaie, 
En somme, la structure juridique afférente à la propriété privée, mise en place par le CCQ et correspondant aux caractéristiques permettant la gestion économique de l'eau, constitue le point d'ancrage fondamental de l'analyse visant à déterminer si la propriété peut s'appliquer à l'eau souterraine. D'ailleurs, les modalités et démembrements de la propriété privée fournissent les seuls cadres juridiques permettant d'explorer la relation entre la propriété commune et l'eau souterraine ${ }^{46}$. Même la propriété publique est liée aux formes de la propriété privée ${ }^{47}$. Finalement, l'appréhension de l'eau souterraine par le droit civil en tant que bien renforce l'importance fondamentale du droit de propriété privée en posant les prémisses d'une dynamique conflictuelle d'appropriation et d'exclusion ${ }^{48}$. En effet, si la ressource se raréfie, son appropriation exclusive à des fins sécuritaires est recherchée ${ }^{49}$. Si la volonté d'utiliser l'eau est entravée, se manifeste une tendance visant à parfaire le droit réel sur le bien afin d'assurer le contrôle sur celui-ci50.

tout vaut tant (tout, sans doute, sauf l'essentiel, mais vous ne pourrez obtenir l'essentiel que parce que tout le reste peut être obtenu par de l'argent). Derrière chaque obligation [...], chaque bien, et même chaque personne (que l'on songe à l'éventualité des accidents corporels), le droit aperçoit d'avance les dommages-intérêts qui pourront les représenter quelque jour, et les dommages-intérêts, ce sont des sommes d'argent."

46. Voir ci-dessous 2.2 et 2.3.

47. Voir notamment art. 915-918 C.c. 3 .

48. Selon David Lametti, "The Concept of Property : Relations through Objects of Social Wealth", (2003) Vol.53, U.T.L.J., 325, aux pp. 334-335 : "[a]t bottom, private property places the ultimate control over some scarce and valuable social resource in the hands of the individual. [...]The most obvious manifestation of vesting control in a single person has been characterized as the ability to exclude others from access to or use of that same resource."

49. Pour une illustration concrète, voir David Aubin, Pierre Cornut \& Frédéric Varone, "Access to water resources in Belgium : strategies of public and private suppliers", (2007) Vol.9, Water Policy, 615, aux pp. 617 et 628-629.

50. Selon Martine Rémond-Gouilloud, "Ressources naturelles et choses sans maîtres», (1985) Vol.2 No5, Recueil Dalloz, 27, aux pp. 28-29, le premier réflexe de la personne confrontée à la pénurie est l'appropriation, ce qui provoque l'extension du domaine de la propriété. 
Devant la force d'attraction de la propriété privée en droit civil québécois, ne subsiste qu'une alternative réelle à la qualification de l'eau souterraine comme bien : la chose commune, ou res communis. Par définition, une res communis n'est pas appropriable. Une chose commune n'est donc pas un bien puisqu'un bien est obligatoirement approprié ou susceptible d'appropriation, publique ou privée 51 . Puisque le fonctionnement du droit civil repose sur le droit de propriété et l'appropriation des biens, la res communis constitue une catégorie juridique qui se soustrait à sa structure et à son fonctionnement en tant que régime de gestion des ressources. Si l'eau souterraine est une res communis, la propriété n'en permet pas la gestion économique.

L'évolution historique $\mathrm{du}$ statut juridique de l'eau souterraine confirme que la propriété privée est étroitement liée à la ressource mais indique aussi que l'eau souterraine pourrait être caractérisée alternativement comme chose commune.

$\mathrm{Au}$ début des années 1970, une réflexion générale sur le statut juridique de l'eau a lieu au Québec dans le cadre de la Commission d'étude des problèmes juridiques de l'eau (ci-après "Commission Legendre") 52 . À cette occasion, un constat s'impose : l'eau souterraine est considérée comme un bien appartenant au propriétaire du sol. Bien que la Commission Legendre n'offre pas de conclusion explicite à cet effet, les commentaires qu'elle émet laissent peu de place à une autre interprétation ${ }^{53}$. De plus, les

51. Biens et propriété, supra note 34, aux pp. 8, 19; Introduction au droit des biens, supra note 37 , p. 62; Droit civil. Les biens, supra note 45 , à la p. 77. Pour une distinction élaborée entre les biens publics et privés d'un côté, et les res communes de l'autre, voir Marie-Alice Chardeaux, Les choses communes, Paris, Librairie Générale de Droit et de Jurisprudence, 2006 (ci-après "Les choses communes"), aux pp. 62-103.

52. Commission d'étude des problèmes juridiques de l'eau, Rapport de la Commission d'étude des problèmes juridiques de l'eau, Québec, Ministère des Richesses naturelles, Gouvernement du Québec, 1975 (ci-après "Problèmes juridiques de l'eaw").

53. Ibid, à la p. 303, qui réfère à l'article 414 du Code civil du Bas Canada (ci-après "C.c.B.-C."), désormais l'article 951 C.c.Q., de même qu'aux décisions Robert c. Fabrique de Montréal, (1898) 4 R.J. 279 (C.S.) et Dame Lortie c. Corporation de l'Ange-Gardien, (1954) C.S. 91. 
(2008) 38 R.D.U.S.

Lagestion économique

de l'eau souterraine

503

par le droit de propriété au Québec

études produites au soutien de la Commission Legendre avancent sans équivoque que l'eau souterraine est un bien appartenant au propriétaire du sol ${ }^{54}$.

À la suite de la Commission Legendre, la doctrine réaffirme à plusieurs occasions que l'eau souterraine appartient au propriétaire du sol55. La jurisprudence confirme ce statut ${ }^{56}$. Jusqu'en 1999, la position du gouvernement du Québec est au même effet 57 .

Cependant, la relation actuelle entre la propriété et les eaux souterraines n'est plus établie aussi clairement. En 1997, débute au Québec une autre réflexion sociale de grande ampleur à propos

54. Jules Brière, Les droits de l'État, des riverains et du public dans les eaux publiques de l'État du Québec, Étude 1.2, Québec, Commission d'étude des problèmes juridiques de l'eau, 1970, aux pp. 2, 5 et 19-20. Quant à lui, Henri Brun, Histoire du droit québécois de l'eau (1663-1969), Étude 1.1, Québec, Commission d'étude des problèmes juridiques de l'eau, 1969, à la p. 41, conclut que la volonté de considérer l'eau comme res communis est constamment mise en échec par le droit positif québécois.

55. Guy Lord \& al., Le droit québécois de l'eau, Centre de recherche en droit public de l'Université de Montréal, Québec, Ministère des Richesses Naturelles, 1977, Vol.1, aux pp. 119-122; René Dussault \& Louis Borgeat, Traité de droit administratif, 2ième éd., Québec, Presses de l'Université Laval, 1986, Vol.2, à la p. 117; Patrick Cossette, "Les eaux souterraines au Québec", (1999) Vol.6, B.D.M., 132, à la p. 141.

56. Gilbert c. Carrière Hébert, J.E. 80-145 (C.P.); Simoneau c. Berthiaume, supra note 39; Jean c. Ferland, [2000] R.D.I. 605 (C.S.). Les motifs de l'affaire Dufour c. Grégoire, B.E. 2004BE-518 (C.Q.) (ci-après "Dufour c. Grégoire»), aux pp. 5-6, semblent aussi reposer sur le principe de propriété des eaux souterraines bien que le tribunal évite de se prononcer explicitement à propos de leur statut juridique.

57. La problématique des eaux souterraines, supra note 23, aux pp. 47-48; Ministère de l'Environnement et de la Faune, Plan d'action pour la mise en ouvre de la Politique de protection et de conservation des eaux souterraines, Projet, Québec, Gouvernement du Québec, 1996, aux pp. 22-23 et 71-74; Ministère de l'Environnement et de la Faune du Québec, Politique de protection et de conservation des eaux souterraines, Document du travail, Québec, Gouvernement du Québec, 1997, à la p. 13; Ministère de l'Environnement, La gestion de l'eau au Québec: document de consultation publique, Québec, Gouvernement du Québec, 1999, aux pp. 12, 18-19. 
de la gestion de l'eau ${ }^{58}$. À cette occasion, l'historique juridique semble encore imposer que l'eau souterraine soit catégorisée $a$ priori comme un bien appartenant au propriétaire du sol, mais une évolution du statut de la ressource est souhaitée et présentée comme inévitable 59 . À la suite d'une consultation publique, la Commission sur la gestion de l'eau au Québec propose, en 2000, la modification du statut juridique de l'eau souterraine afin qu'elle devienne une res communis :

Actuellement, les eaux souterraines et les eaux de surface ont un statut juridique différent. Les premières constituent un bien de propriété privée lié à la propriété immobilière et les secondes ont un statut de bien commun[...] Il ne s'agirait pas de procéder à une expropriation ni à une nationalisation des eaux souterraines, mais 'seulement une abolition du droit de propriété sur la chose commune'[...] En d'autres termes, le statut des eaux souterraines viendrait rejoindre celui déjà attribué aux eaux de surface. 60

Cette recommandation rejoint alors les voux de certains auteurs et correspond à l'opinion publique prévalente ${ }^{61}$. En 2002,

58. Gouvernement du Québec, Symposium sur la gestion de l'eau au Québec. Document de référence, Québec, Gouvernement du Québec, 1997, p. I (Préambule du Premier ministre Lucien Bouchard).

59. Lorne Giroux, "Le droit de l'eau : à qui appartient l'eau", 81, aux pp. 8384, dans Jean-Pierre Villeneuve, Alain Rousseau \& Sophie Duchesne, dir., Symposium sur la gestion de l'eau au Québec. Actes du Symposium. L'état de l'eau au Québec, Sainte-Foy, INRS-Eau, 1998 (Vol.2); Louise Ouellet \& Claude Laurent, "L’état de l'eau au Québec. Le statut juridique" dans Jean-Pierre Villeneuve, Alain Rousseau \& Sophie Duchesne, dir., Symposium sur la gestion de l'eau au Québec. Actes du Symposium. L'état de l'eau au Québec, Sainte-Foy, INRS-Eau, 1998 (Vol.2) 257 à la p. 262.

60. Commission sur la gestion de l'eau au Québec, L'eau, ressource à protéger, à partager et à mettre en valeur, Québec, Bureau d'audiences publiques sur l'environnement, 2000 (ci-après "L'eau ressource à protégen»), Tome I, à la p. 121.

61. Voir Madeleine Cantin Cumyn, Michelle Cumyn \& Claire Skrinda, "L'eau, chose commune : un statut juridique à confirmer", (2000) 79 R. du B. can. 398 (ci-après "L'eau, chose commune"), aux pp. 401-407 et 409-410, de même que L'eau ressource à protéger, supra note 60, Tome II à la p. 36. 
(2008) 38 R.D.U.S.

La gestion économique

de l'eau souterraine

505

par le droit de propriété au Québec

le gouvernement du Québec prend acte des recommandations relatives à la modification du statut juridique de l'eau souterraine et reconnaît que la ressource est une res communis dans la Politique nationale de l'eau'62. Le gouvernement du Québec s'engage à réviser l'encadrement juridique de l'eau'63. Selon la doctrine, la révision du cadre juridique afin de confirmer le statut de l'eau souterraine comme res communis requiert effectivement la modification des dispositions pertinentes ${ }^{64}$. Or, une telle modification n'est pas encore réalisée65. Ainsi, selon certains auteurs, il semble toujours possible de considérer l'eau souterraine comme un bien appartenant au propriétaire du sol ${ }^{66}$. D'autres renouvellent l'appel en faveur d'une modification des

62. Ministère de l'Environnement, L'eau. La vie. L'avenir. La politique nationale de l'eau, Québec, Gouvernement du Québec, 2002 (ci-après "Politique nationale"), aux pp. 15-17. Notons qu'à la page 15 de la Politique nationale, les eaux souterraines sont identifiées comme patrimoniales. Or, le patrimoine n'a pas d'emprise sur les choses communes : Introduction au droit des biens, supra note 37, aux pp. 1617. Cette contradiction apparente est résolue par la notion de patrimoine commun : voir Marie-José Del Rey, "La notion controversée de patrimoine commun", (2006) No6, Recueil Dalloz, 388 (ci-après "Patrimoine commun"), aux pp. 388-389. Au sujet de l'eau souterraine comme patrimoine commun, voir ci-dessous 2.3 .

63. Voir la Politique nationale, supra note 62, à la p. 92.

64. Pierre Coderre \& Michel Blais, "L'eau vous interpelle, suivez le courant!» dans Service de la formation permanente du Barreau du Québec, Développements récents en droit de l'environnement (2000), Cowansville, Éditions Yvon Blais, 2000, à la p. 19.

65. Selon l'article $1 \mathrm{du}$ Projet de loi $n^{\circ} 92$, l'eau souterraine ne pourrait être appropriée autrement que lorsque la loi, et notamment le C.c.Q., le permet parce que la ressource appartiendrait au patrimoine commun de la nation. Ainsi, il est probable que la sanction du Projet de loi no92 ne modifie pas l'appréhension de l'eau souterraine par le droit civil. Madeleine Cantin-Cumyn \& Sylvie Paquerot, Sortir le projet de loi sur l'eau de l'ambigüité, 18 juin 2008, Gaia Presse (disponible en ligne le 1 novembre 2008 à l'adresse suivante: http://www.gaiapresse. $\mathrm{ca} / \mathrm{fr} /$ analyses/index.php?id=14) soulignent aussi l'ambiguïté de l'article 1 du Projet de loi no92 parce qu'il ne permet pas de prétendre à une réelle modification du statut juridique de l'eau souterraine.

66. Biens et propriété, supra note 34, à la p. 184. 
dispositions pertinentes ${ }^{67}$. La jurisprudence fait état des hésitations relatives au statut de l'eau sans tirer de conclusion ${ }^{68}$.

Puisqu'un survol de la jurisprudence et de la doctrine relatives au statut juridique de l'eau souterraine entretient l'ambiguïté, il est nécessaire de procéder à une analyse détaillée pour déterminer si la propriété s'applique effectivement à la ressource, rendant ainsi possible sa gestion économique.

\section{LA PROPRIÉTÉ ET L'EAU SOUTERRAINE}

Les considérations précédentes requièrent de procéder à l'étude de la relation entre l'eau souterraine d'une part, et la propriété privée, la propriété commune, la propriété publique et le statut de chose commune d'autre part. Cette partie vise à démontrer que l'eau souterraine n'est jamais l'objet de quelque forme de propriété que ce soit. Ainsi, il est d'abord établi que la propriété privée ne peut pas s'appliquer à l'eau souterraine (2.1.), puis que le droit civil ne permet pas la gestion de l'eau souterraine en propriété commune (2.2.), ensuite que l'État n'est pas propriétaire de la ressource (2.3.), et finalement que l'eau souterraine est une res communis (2.4.).

\subsection{La propriété privée et l'eau souterraine}

Selon la jurisprudence et la doctrine, le propriétaire du sol peut être perçu comme le propriétaire des eaux souterraines. Cette assertion repose sur une perception statique qui fait abstraction de la réalité hydrogéologique mais aussi des contraintes issues de l'expression juridique de la propriété immobilière. La fluidité matérielle de la ressource et l'impossibilité d'en borner les contours posent des problèmes de cohérence insolubles pour le

67. Bilan de la réforme, supra note 42, aux pp. 330-331.

68. Lambton (Municipalité de) c. Stanscia, J.E. 2007-295 (C.S.) (ci-après "Lambton c. Stanscia»), aux pp. 6-7; Gauthier c. Côté, 3 avril 2003, Cour supérieure, juge Yves Tardif, 455-05-000712-021 (ci-après "Gauthier c. Côtén), aux pp. 7-8. 
(2008) 38 R.D.U.S.

La gestion économique

de l'eau souterraine

par le droit de propriété au Québec

droit de propriété69. La présente sous-partie constitue un effort de plus pour mettre fin à "l'étrange pérennité de la propriété sur les eaux souterraines", qui semble perdurer en dépit du droit actuel et de la réalité hydrologique ${ }^{70}$.

D'emblée, le droit de propriété permet difficilement l'appréhension de la ressource. La solution initiale proposée par le droit québécois repose sur la propriété privée du sol, qui emporte en principe celle du dessous selon le $\mathrm{CCQ}^{71}$. Le sol sous la surface d'un terrain de même que l'eau souterraine qu'il contient appartiendraient au propriétaire du terrain. L'eau souterraine à l'état naturel devrait alors être considérée comme un bien immobilier ${ }^{72}$. La seule exception possible serait celle des biens

69. Bilan de la réforme, supra note 42 , à la p. 330.

70. Les guillemets réfèrent au titre d'un article de Valérie Varnerot, "L'étrange pérennité du droit de propriété sur les eaux souterraines», (2002) No2, R.J.E., 135 (ci-après "L'étrange pérennité").

71. Article 951 C.c.Q. Le droit de propriété est complété par le droit d'aménager dans le sol des constructions et ouvrages, sous réserve des droits publics sur les nappes d'eau souterraines.

72. Selon l'article 899 C.c. , l'eau souterraine serait obligatoirement un bien meuble ou un bien immeuble. L'article 900 C.c.Q. énonce qu'est immeuble tout ce qui fait partie intégrante d'un fonds de terre jusqu'à en être extrait ou séparé, tandis qu'en vertu de l'article 901 C.c.Q. font partie intégrante d'un immeuble les meubles qui sont incorporés à l'immeuble, qui perdent leur individualité et assurent l'utilité de l'immeuble. L'eau souterraine pourrait être un pur bien immeuble par nature puisque c'est le cas des minéraux dans le sol : Biens et propriété, supra note 34 , à la p. 33. En effet, il serait malvenu de différencier le statut de l'eau souterraine de celui d'autres minéraux liquides notamment la saumure - à partir de la concentration de certains composants chimiques dans ces matières. Le Règlement sur le pétrole, le gaz naturel, la saumure et les réservoirs souterrains, R.Q., c. M-13.1, r.0.3, identifie d'ailleurs les réservoirs de saumure comme des "aquifères". Si cette caractérisation n'était pas acceptée, l'eau souterraine pourrait aussi être un bien immeuble à titre de bien meuble intégré à un immeuble. Une étude des critères qui permettent de déterminer s'il y a intégration fait naître une foule d'arguments en faveur autant qu'en défaveur de la reconnaissance de l'eau souterraine comme immeuble par nature parce que meuble intégré : au sujet des critères, voir Biens et propriété, supra note 34, aux pp. 36-37. Finalement, la ressource pourrait être immeuble par attache ou réunion selon l'article 903 C.c.Q : pour les critères qui permettent de déterminer s'il y a attache ou réunion, 
meubles par anticipation ${ }^{73}$. Cette exception s'appliquerait de façon limitée aux seuls cas où l'eau souterraine fait spécifiquement et explicitement l'objet d'un acte de disposition ${ }^{74}$.

de même que la distinction avec les immeubles par intégration, voir Biens et propriété, supra note 34, aux pp. 42-48. Cependant, ces distinctions sont pertinentes uniquement à des fins théoriques parce que les règles du droit immobilier s'appliqueraient généralement à l'eau souterraine quelle que soit sa caractérisation exacte parmi les biens immobiliers : voir Biens et propriété, supra note 34, aux pp. 39-42.

73. Art. 900 C.c.Q.

74. Un exemple de la caractérisation des eaux souterraines comme bien meuble est offert par l'affaire Simoneau c. Berthiaume, supra note 39, aux pp. 376-378. La dissidence souligne ainsi les difficultés qu'une telle caractérisation soulève : “j’ai beaucoup de difficulté à concevoir qu'on puisse être propriétaire d'une source sans être propriétaire du fonds de terre ou elle jaillit; après tout 'la propriété du sol emporte la propriété du dessus et du dessous'[...] Si l'on retient la prétention des appelants, faudra-t-il conclure qu'ils sont également propriétaires de la nappe phréatique qui alimente cette source? Si l'eau jaillit à un autre endroit, à proximité, pourra-t-on prétendre qu'in s'agit d'une même source? Et, si le vendeur a conservé la propriété de cette source, la propriétaire actuelle du terrain [...] y aurait-elle accès et pourrait-elle s'y alimenter? Si cette source doit être exclue de la propriété du fonds - et je reviendrai sur cette question -, dans quel rayon doit-elle l'être puisque cette source n'est décrite ni par tenant ni par aboutissants (art. 2168 C.C.)? Et comment les 'propriétaires de la source' pourraient-ils y avoir accès puisqu'ils ne bénéficient d'aucun droit de passage pour s'y rendre?" (Citations omises) La critique minoritaire de la Cour d'appel correspond à la solution jurisprudentielle française selon laquelle il est impossible de concevoir l'eau souterraine comme un bien mobilier: L'étrange pérennité, supra note 70 , à la p. 146. La solution française semble raisonnable, puisque l'eau souterraine considérée comme individualité juridique distincte du sol n'est plus de l'eau souterraine. La propriété d'un bien meuble implique que sa possession soit possible. Or, l'eau souterraine migre naturellement hors des limites d'une propriété immobilière, de telle sorte que sa possession effective et durable est impossible : voir infra note 87 . Pour réellement posséder un volume d'eau souterraine comme bien meuble, il faut le pomper ou l'enfermer dans un ouvrage, ce qui implique automatiquement que l'eau ainsi capturée ne soit plus souterraine. Quoi qu'il en soit, puisque la caractérisation des eaux souterraines à l'état naturel comme bien meuble par anticipation représente un cas exceptionnel, ses répercussions ne sont pas traitées. 
Le principe de propriété immobilière des eaux souterraines pose plusieurs problèmes. D'abord, il repose sur la prémisse que celui qui possède un terrain est propriétaire de l'eau souterraine contenue dans le volume créé par la projection des frontières de la propriété vers le centre de la Terre. A priori, le volume d'eau souterraine possédé est donc fini. Cependant, il est accru par la recharge des eaux souterraines provenant de l'infiltration des précipitations dans le sol. La pluie qui est considérée traditionnellement comme bien mobilier sans propriétaire, ou res nullius, se joint en partie aux eaux souterraines et devient objet de propriété par le mécanisme de l'occupation ${ }^{75}$. Si le volume d'eau souterraine soumis à la propriété s'accroît avec le temps selon la pluviométrie, il impose néanmoins des limites quantitatives maximales précises aux activités de captage par le propriétaire du sol. De façon schématique, la quantité maximale qui peut être captée correspond au volume initial de la ressource contenue dans le sol lors de l'acquisition du titre par son propriétaire, additionné du volume de précipitations tombées depuis ce moment à la surface du terrain et infiltrées dans le sol.

Non seulement cette limite n'est jamais prise en compte par le droit civil, mais son dépassement empêche l'application cohérente du principe de propriété des eaux souterraines par la propriété du sol. En effet, un bien immobilier est toujours enclavé par d'autres biens immobiliers sur lesquels s'exercent des droits de propriété qui justifient des titres équivalents sur les eaux souterraines voisines ${ }^{76}$. Compte tenu de la perméabilité des sols et du gradient hydraulique provoqué par une activité de captage, il est inévitable que l'eau souterraine captée au-delà de la limite quantitative maximale constituée du volume de la propriété en

75. Articles 934, 935 et 948 C.c.Q.; Denys-Claude Lamontagne, "Règles particulières à la propriété immobilière et servitudes" dans Barreau du Québec \& Chambre des notaires du Québec, La réforme du code civil. Personnes, successions et biens, Vol.1, Québec, Presses de l'Université Laval, 1993, 517 à la p. 524 .

76. Il existe toujours une propriété voisine : art. 915, 918 et 936 C.c.Q; Pierre Labrecque, Le domaine public foncier au Québec, Cowansville, Éditions Yvon Blais, 1997 (ci-après "Le domaine public foncien"), à la p. 7; Biens et propriété, supra note 34, aux pp. 9-14. 
sous-sol additionné de la recharge pluviale appartienne originellement à un propriétaire voisin ${ }^{77}$. Le voisin peut alors argumenter qu'un enrichissement injustifié s'est produit et réclamer compensation de la part du capteur ${ }^{78}$. De plus, si la prémisse de départ sur laquelle repose la propriété de la ressource autant que la mécanique du déplacement des eaux souterraines dans le sol sont respectés, il appert que l'enrichissement injustifié se produit bien avant le captage des eaux souterraines: une quantité d'eau souterraine qui est située sous la propriété d'un voisin, qui appartient au voisin, et qui se trouve dans l'aire d'appel de l'ouvrage de captage, entre dans les limites souterraines du terrain sur lequel s'exercent les activités de captage dès le début de ces activités et peut-être plusieurs années avant le captage effectif.

Ensuite, le captage de l'eau souterraine par le propriétaire du sol change le patron d'écoulement naturel dans le sol et crée un rabattement de la surface de la nappe phréatique qui peut s'étendre au-delà des frontières de sa propriété79. Il est possible que les propriétaires voisins doivent modifier leurs propres puits de captage pour conserver l'usage de leurs eaux souterraines. Dans ce cas, l'usage d'un objet de propriété change l'usage qu'un autre propriétaire peut faire du sien ${ }^{80}$. Une telle situation peut être

77. Voir Jacques Sironneau \& al., Lamy environnement, l'eau, Paris, Lamy, 1996- (mises à jour périodiques) (ci-après "Lamy eaw"), II112-49.

78. Art. 1493-1496 C.c.Q.

79. Lamy eau, supra note 77, II112-49 et II112-50.

80. La doctrine américaine reconnaît les problèmes liés à la définition des droits de propriété corrélatifs entre plusieurs capteurs d'eau souterraine et les explique par trois facteurs: "First, one pumper's [use] seldom precludes access to the necessary amount of water: instead, pumping increases the cost of access. Second, all pumpers are responsible for diminished access. Third, groundwater [use] must be integrated with surface rights because ground and surface systems are often hydrologically connected.", dans Dan Tarlock, Law of water rights and resource, Eagan, Thompson West, 1988 -(mises à jour périodiques), §6:8. Aux États-Unis, la reconnaissance du droit de propriété sur l'eau souterraine par le biais de la propriété du sol a débouché sur un phénomène identifié comme la "loi de la plus grosse pompe". Il s'agit d'une dynamique par laquelle l'accroissement des usages abaisse la 
(2008) 38 R.D.U.S.

La gestion économique

de l'eau souterraine

par le droit de propriété au Québec

gérée par l'obligation de bon voisinage, qui reconnaît la possibilité de certaines interférences entre des droits de propriété exercés normalement ${ }^{81}$. Mais le cas de l'assèchement d'un puits voisin à cause de l'aménagement d'un ouvrage de captage se trouve hors de portée des règles de bon voisinage lorsque les deux puits sont utilisés à des fins strictement domestiques et que des sources alternatives ne sont pas disponibles. Dans une telle situation, les inconvénients sont à la fois normaux puisque le puits nouvellement aménagé permet de subvenir au besoin vital d'alimentation en eau d'un ménage, mais aussi intolérables parce qu'ils privent un autre ménage de sa source d'eau potable ${ }^{82}$. Ces interférences induisent des distorsions dans le caractère exclusif de la propriété susceptibles de miner les fondements de ce droit lorsqu'il s'applique aux eaux souterraines ${ }^{83}$.

surface de la nappe phréatique et la rend plus difficile d'accès, favorisant ainsi celui qui dispose des plus importants moyens techniques et financiers pour capter l'eau au détriment des autres usagers, dont l'accès à la ressource diminue ou s'éteint. Pour un aperçu du contexte juridique dans lequel s'est produit ce phénomène, voir ibid, §4:7-§4:10.

81. Art. 976 C.c.Q.

82. Voir L'étrange pérennité, supra note 70, aux pp. 147-150, 156-158.

83. Les droits d'exploitation corrélatifs sur une ressource liquide dans le sol ne semblent pas avoir fait l'objet d'une réflexion autre qu'embryonnaire en doctrine québécoise, faute de nécessité puisque le Québec n'est pas riche en hydrocarbures. À propos du pétrole, Patrick Kenniff, "Le contrôle de l'utilisation du sol et des ressources en droit québécois", (1975) 16 C. de D. 763 à la p. 804 et (1976) 17 C. de D. 85, à la p. 164, note à la fois que de tels droits d'exploitation corrélatifs menacent le droit de propriété et qu'ils doivent être réglementés étroitement pour assurer la conservation de la ressource et une exploitation optimale. Toutefois, le législateur élude le problème puisque le régime de la Loi sur les mines, L.R.Q., c. M-13.1 (ci-après "LM") ne semble envisager qu'un droit d'exploitation unique sur un réservoir souterrain : voir notamment les articles 194, 195 et 196 LM. Notons que le droit minier extra-provincial traite de la question par le biais de la "règle de capture", qui offre au titulaire du droit minier l'appropriation de la ressource lors $\mathrm{du}$ recouvrement de la substance minérale. En fait, cette règle provient initialement du droit américain applicable aux eaux souterraines. Pour éviter une course à l'extraction entraînant un surinvestissement et une exploitation sous-optimale, la règle de capture a été profondément modifiée par l'imposition de règles d'espacement et d'،accords de mise en commun": à ce sujet, voir Nigel Bankes, "Pooling Agreements in 
En somme, l'appropriation des eaux souterraines néglige les liens qui lient l'objet du droit au milieu matériel et juridique dans lequel il se situe. L'atténuation de l'absolutisme exclusif de la propriété sur la ressource n'étant plus possible par l'intermédiaire des mécanismes juridiques en place, la propriété privée des eaux souterraines doit être remise en cause.

Par ailleurs, les eaux souterraines sont en transit dans le sol. Au cours de tout laps de temps, une certaine quantité d'eau souterraine quitte un terrain tandis qu'une autre quantité y pénètre. Le droit de propriété ne s'exerce donc jamais sur les mêmes eaux souterraines. Le droit ne dispose pas d'instrument adéquat pour gérer de façon cohérente le passage d'un patrimoine à l'autre lorsque l'eau souterraine passe d'un terrain à l'autre. Cette migration soulève des problèmes regroupés sous deux aspects interdépendants : 1) l'entrée de l'eau souterraine dans le patrimoine d'un propriétaire lors de sa migration dans le sol qu'il possède; 2) la sortie de l'eau souterraine du patrimoine d'un propriétaire lors de sa migration hors du sol qu'il possède.

Quant à l'entrée de l'eau souterraine dans le patrimoine, la ressource peut a priori être acquise par contrat, par succession, par occupation, par prescription, ou par accession ${ }^{84}$. L'application

Canadian Oil and Gas Law», (1994) 33 Alta. L. Rev. 493, aux pp. 497502. Ainsi, la propriété privée est aussi incapable de gérer la ressource fluide en sous-sol dans le domaine minier.

84. Voir Pierre-Claude Lafond, Précis de droit des biens, Montréal, Éditions Thémis, 1999 (ci-après "Précis de droit des biens"), aux pp. 987-988. Selon les dispositions de l'article 916 C.c.Q, l'acquisition peut aussi se faire par tout autre mode que la loi prévoit. En vertu de l'article 41 de la Loi sur la qualité de l'environnement, L.R.Q., c. Q-2 (ci-après "LQE»), toute municipalité peut, avec l'autorisation du ministre du Développement durable, de l'Environnement et des Parcs (ci-après "Ministre"), exproprier des sources d'approvisionnement d'eau et autres immeubles ou droits réels situés en dehors de son territoire et requis pour l'installation d'un système d'aqueduc ou d'une usine de traitement des eaux ou pour l'installation ou la protection d'une prise d'eau d'alimentation. L'article $42 \mathrm{LQE}$ prévoit un pouvoir similaire pour un exploitant privé. Bien que la jurisprudence n'ai pas étudié spécifiquement la question, l'article $41 \mathrm{LQE}$ est implicitement interprété comme visant l'expropriation d'un terrain et 
(2008) 38 R.D.U.S.

La gestion économique

de l'eau souterraine

par le droit de propriété au Québec

de chacun de ces modes d'acquisition se bute à d'importants obstacles. Premièrement, le contrat et la succession ne sont pas des modes d'acquisition pertinents pour appréhender la migration progressive de l'eau souterraine dans le sol ${ }^{85}$.

Deuxièmement, l'occupation n'est possible que dans le cas des biens meubles sans propriétaire et elle implique la possession ${ }^{86}$. Or, l'eau souterraine est généralement un immeuble approprié qui échappe à la possession du propriétaire du terrain tant qu'elle n'est pas captée, c'est-à-dire tant qu'elle est souterraine ${ }^{87}$. L'occupation ne permet donc pas l'acquisition de la ressource, à moins d'accepter une modification de la prémisse de départ selon laquelle la propriété du sol entraîne la propriété de l'eau souterraine.

Troisièmement, l'acquisition du droit de propriété sur les eaux souterraines par union grâce au droit d'accession est problématique. En effet, aucun des deux types d'accession possibles ne peut s'appliquer aux eaux souterraines : l'accession

non celle de l'eau souterraine : voir notamment Lac-Mégantic (Ville de) c. Lapierre, J.E. 2004-2033 (C.S.), aux pp. 3-4, et Brousseau \& Fils ltée c. Chandler (Ville de), B.E. 98BE-1290 (C.S.), à la p. 3.

85. Cette affirmation est incontestable pour la succession. Par ailleurs, la pratique juridique québécoise ne connaît pas de contrat entre voisins transférant la propriété de l'eau souterraine au gré de son écoulement naturel. Notons qu'il existe en France des contrats de nappe, mais ils visent principalement la police des usages au plan qualitatif et ne reposent pas sur la propriété : Philippe Billet, "La régulation juridique des conflits liés à la gestion des eaux souterraines", (2001) 3 R.J.E. 401 à la p. 415.

86. Art. 914, 935 C.c.Q.; Précis de droit des biens, supra note 84, aux pp. 995-996.

87. Dans le cadre de la propriété, la possession doit avoir les caractéristiques de ce droit, c'est-à-dire être exclusive, absolue et perpétuelle : article 921 C.c.Q. Or, les eaux séjournent en moyenne environ 13 ans dans le sol lors de leur migration avant de retourner de façon naturelle aux rivières et aux océans : Olivier Banton, "Le capital eau : son potentiel et ses usages. Les eaux souterraines" dans Jean-Pierre Villeneuve, Alain Rousseau \& Sophie Duchesne, dir., Symposium sur la gestion de l'eau au Québec. Actes du Symposium. L'état de l'eau au Québec, Sainte-Foy, INRS-Eau, 1998 (Vol. 2) , 45 à la p. 47. 
artificielle vise les constructions, ouvrages ou plantations, alors que l'accession naturelle, qui est involontaire et correspond mieux à la réalité hydrologique de la ressource, ne vise que des cas spécifiques et exceptionnels relatifs aux eaux de surface88. De plus, l'eau souterraine qui migre dans un terrain appartient $a$ priori à un voisin. À prime abord, la prescription semble plus appropriée que l'acquisition.

Quatrièmement, la prescription ne constitue pas non plus un mécanisme juridique adéquat pour permettre l'appropriation de la ressource. Restant unies au sol, les eaux souterraines qui passent d'une propriété immobilière à l'autre restent des biens immobiliers. Alors, les conditions de validation de la prescription acquisitive décennale en matière immobilière doivent s'appliquer, ce qui débouche sur une situation ingérable89. De plus, les caractéristiques du sol et les aléas du découpage de sa surface par les lignes de propriété peuvent permettre une migration rapide de l'eau souterraine d'une propriété à l'autre, notamment en cas de captage, ce qui rend difficile l'application de délais de prescription acquisitive rigides ${ }^{90}$.

Quant à la sortie de l'eau du patrimoine, le processus par lequel les eaux souterraines quittent les frontières du terrain et se soustraient au droit de propriété n'est pris en charge par aucun mécanisme juridique. En principe, la propriété n'est pas provisoire et ne se perd pas par le non-usage ${ }^{91}$. Il est possible de se départir de la propriété en disposant d'un bien de deux façons : juridiquement ou matériellement ${ }^{92}$. La disposition juridique, telle

88. Art. 954-970 C.c.Q.

89. La demande en justice prévue à l'article 2918 C.c.Q. constitue un exemple de ces conditions.

90. Art. 2917 C.c.Q. Comment le droit de propriété et le mécanisme de prescription acquisitive peuvent-ils gérer la situation suivante : une eau de pluie tombe sur un terrain où elle s'infiltre, puis migre vers la propriété voisine où elle est captée et embouteillée cinq ans après être tombée. L'eau embouteillée par le voisin peut-elle faire l'objet d'une revendication (art.912 et 953 C.c.Q)?

91. Précis de droit des biens, supra note 84, aux pp. 275-277.

92. Biens et propriété, supra note 34, aux pp. 154-166. 
(2008) 38 R.D.U.S.

La gestion économique

de l'eau souterraine

par le droit de propriété au Québec

l'aliénation, n'est pas applicable aux eaux souterraines qui migrent naturellement à l'insu du propriétaire et sans son intervention. La disposition matérielle par rénovation, altération, ou destruction est aussi inapplicable dans le cas de la migration des eaux souterraines. D'ailleurs, si l'eau souterraine était un objet de propriété et s'écoulait dans la propriété d'un voisin, elle continuerait d'appartenir à son propriétaire, et le voisin devrait en permettre la recherche ou l'enlèvement ${ }^{93}$. En principe, le droit existe aussi longtemps que l'objet sur lequel il porte ${ }^{94}$. Il est impossible de présumer l'abandon d'un bien ${ }^{95}$. Ainsi, en tentant d'appliquer le droit de propriété aux eaux souterraines sans qu'un mécanisme juridique ne rende compte de la migration de la ressource hors du patrimoine du propriétaire, le caractère perpétuel de la propriété est remis en cause.

En somme, la propriété privée est incapable de fournir un cadre juridique aux eaux souterraines. Son application à la ressource provoque des incohérences dans la structure du droit civil. Un autre artefact juridique doit être identifié pour tenter d'établir un lien entre la propriété et l'eau souterraine.

\subsection{La propriété commune : modalités et démembrements de la propriété privée}

Compte tenu des problèmes d'application de la propriété privée aux eaux souterraines, l'appréhension de la ressource par le biais des modalités et des démembrements du droit de propriété a été envisagée afin de lui trouver un statut juridique séant. La copropriété indivise et le droit d'usage, qui ont tous deux fait l'objet d'une attention particulière, peuvent être considérés comme

93. Art. 989 C.c.Q.

94. Sylvio Normand, "La notion de modalité de la propriété " dans Sylvio Normand, Mélanges offerts au professeur François Frenette: Étude portant sur le droit patrimonial, Québec, Presses de l'Université Laval, 2006, 255 à la p. 258.

95. Introduction au droit des biens, supra note 37 à la p. 38. De plus, l'abandon d'un droit réel immobilier que l'on désire rendre opposable aux tiers doit donner lieu à une inscription au registre foncier : art. 2938 al. 1 C.c.Q. 
des cadres juridiques de gestion apparentés à la propriété commune de la ressource. Or, ni l'une ni l'autre ne permettent effectivement la gestion de l'eau souterraine.

D'abord, la doctrine établit que la copropriété par indivision, qui est une modalité de la propriété, constitue une forme potentielle de propriété commune de l'eau souterraine en droit civil québécois à cause de l'unicité de la ressource et des influences que les usages exercent les uns sur les autres ${ }^{96}$. Les conséquences de l'application de ce cadre juridique à la ressource sont ainsi détaillées :

En premier lieu, 'chaque indivisaire peut se servir du bien indivis, à condition de ne porter atteinte ni à sa destination ni aux droits des autres indivisaires' (art.1016 C.c.Q.). En second lieu, 'les indivisaires administrent le bien en commun' (art.1025 C.c.Q.). En troisième lieu, 'les décisions visant à aliéner le bien indivis, à le partager, à le grever d'un droit réel, à en changer la destination ou à y apporter des modifications substantielles sont prises à l'unanimité' (art.1026 C.c.Q.). ${ }^{97}$

Cependant, l'application de la copropriété indivise à l'eau souterraine soulève des problèmes incontournables. Premièrement, le partage de la propriété indivise peut généralement être demandé98. Cependant, l'eau souterraine est techniquement indivisible. A priori, ce problème peut être évité en présumant que le partage de la copropriété indivise de l'eau souterraine est rendu impossible à cause de l'affectation de la ressource à un but durable ${ }^{99}$. Toutefois, il est douteux que toutes les utilisations de l'eau souterraine soient à des fins durables.

96. L'eau, chose commune, supra note 61 à la p. 404.

97. Ibid. aux pp. 404-405.

98. Voir l'art. 1030 C.c.Q.

99. Ibid. Il est possible de s'inspirer de l'affaire Rousseau c. Lafrance, J.E. 2007-401 (C.S.), dans laquelle le partage d'un puits artésien qui fait l'objet d'une copropriété par indivision est jugé impossible parce qu'affecté à un but durable, c'est-à-dire l'alimentation en eau domestique. 
(2008) 38 R.D.U.S.

La gestion économique

de l'eau souterraine

par le droit de propriété au Québec

Deuxièmement, la taille, les limites et les caractéristiques des aquifères sont généralement inconnues ${ }^{100}$. Par ailleurs, les eaux souterraines sont reliées aux eaux de surface, tels les rivières et lacs. Il n'est donc pas possible de déterminer exactement qui sont les copropriétaires indivis de la ressource. De plus, leur nombre est potentiellement très élevé. Or, les coûts de négociation augmentent de façon exponentielle plus le nombre de propriétaires indivis croît ${ }^{101}$. Troisièmement, il n'existe pas de règle qui puisse déterminer la part de la ressource pour chacun des copropriétaires ${ }^{102}$. Une telle situation où les droits quantitatifs sont mal définis entraîne la spoliation de la ressource103. En somme, la copropriété indivise n'offre pas un cadre juridique effectif permettant la gestion de l'eau souterraine.

Par ailleurs, le droit d'usage, qui est un démembrement de la propriété, peut être relié à la propriété commune parce qu’il permettrait un nombre illimité d'utilisateurs de l'eau souterraine s'il constituait le cadre juridique de gestion de la ressource104. En

100. A Science Case Study, supra note 24 aux pp. 5 et 10-11.

101. Voir William Blomquist, "Rôle de la propriété commune dans le gestion des ressources en eau" dans Max Falque \& Michel Massenet, dir., Droits de propriété, économie et environnement. Les ressources en eau, Paris, Dalloz, 2000, 227. Ce constat repose sur les arguments initialement avancées par Ronald Coase, "The problem of social cost", (1960) 3 J. L. \& Econ. 1.

102. Voir L'eau, chose commune, supra note 61 à la p. 405. Faut-il appliquer la présomption de parts égales? Les parts respectives de la ressource pourraient être établies en proportion des superficies des terrains, de la composition du sol, du volume d'eau situé sous chaque terrain, de la pluie reçue par chaque terrain, chacune de ces hypothèses ayant du mérite selon que l'aquifère soit très étendu, que le coefficient de perméabilité des sols varie, ou que les terrains soient pavés et drainés en tout ou en partie.

103. Voir Karin Kemper, "Rethinking groundwater management" dans Caroline Figuáeres, Johan Rockström, Cecilia Tortajada, dir., Rethinking water management: innovative approaches to contemporary issues, London, Earthscan Publications, 2003, 120 à la p. 135. Il s'agit d'une dynamique apparentée à la tragédie des biens communs identifiée par Garrett Hardin, "The Tragedy of the Commons", (1968) 162 Science 1243.

104. Il s'agit du droit d'usage au sens des art. 1172-1176 C.c.Q. La Commission Legendre a étudié le prédécesseur de l'art. 1172 C.c.Q., soit 
effet, le droit d'usage ne permet pas de porter atteinte à la substance de la chose qui en est l'objet, de telle sorte que des usages successifs pourraient s'enchaîner indéfiniment sans variation significative de la qualité et de la quantité de l'eau. Cependant, l'utilisation de l'eau a généralement pour effet d'en dégrader la qualité au point de la rendre inutilisable à certaines fins ${ }^{105}$. De plus, le droit d'usage est un droit réel qui porte sur la propriété d'autrui106. Or, l'eau souterraine n'est jamais objet de propriété107. Par ailleurs, le droit d'usage se limite aux besoins de son titulaire et à ceux de sa famille. Dans ce cas, l'utilisation de l'eau à des fins autres et dans des quantités plus importantes que celles liées aux fins domestiques ne serait pas possible. Finalement, la pertinence du droit d'usage à l'égard des eaux souterraines est limitée dans la mesure où celles-ci ne peuvent faire l'objet d'un usage tant qu'elles sont dans le sol, c'est-à-dire tant qu'elles sont souterraines ${ }^{108}$.

l'art. 487 C.c.B.-C. : Problèmes juridiques de l'eau, supra note 52, aux pp. 75 et 335. Notons que la Commission Legendre a suggéré l'emploi du terme "utilisation" plutôt qu' "usage" dans les situations qui ne visent pas spécifiquement le droit d'usage tel que défini par le C.c.Q. pour éviter certains glissements sémantiques. Ici, cette convention n'est pas respectée parce que la littérature relative à la gestion des eaux consacre le terme d'usage (ex. : "conflits d'usages") et que le C.c.Q. induit la confusion lui-même (ex. : art. 913, 920, 980, 982 et 1172 C.c.Q.).

105. À l'occasion, il est intéressant de souligner que c'est le processus de dégradation d'une qualité ou d'une caractéristique de l'eau qui donne une valeur à l'eau. Le contrôle de l'eau est recherché pour lui faire subir ce processus de dégradation, quelle que soit sa forme spécifique. L'eau qui ne subit pas ce processus n'a aucune utilité. Une eau qui ne peut jamais être utilisée à l'avenir, c'est-à-dire qui ne peut jamais subir ce processus de dégradation, n'a aucune valeur. C'est l'utilisation potentielle qui peut en être faite à l'avenir, par le fait même sa dégradation éventuelle, qui donne une valeur à l'eau. Lorsqu'elle est appréhendée par le droit de propriété (sous forme d'eau embouteillée par exemple), l'eau est donc un bien consomptible.

106. Art. 1172 C.c.Q.

107. Voir section 2.4 du texte.

108. Cependant, il est possible d'envisager un usage passif de la ressource à l'état naturel : l'eau souterraine contribue au volume du sol et empêche son affaissement. L'affaissement du sol menace l'intégrité des bâtiments. Par exemple, dans Commission de la Capitale nationale c. Pugliese, [1979] 2 R.C.S. 104, la Cour se penche sur la question suivante : le propriétaire 
Ainsi, les modalités et les démembrements de la propriété ne permettent pas une appréhension juridique satisfaisante des eaux souterraines ${ }^{109}$. La copropriété indivise et le droit d'usage ne constituent pas des cadres juridiques effectifs de gérance de la ressource. Il est possible de conclure que la propriété commune est inapplicable à l'eau souterraine au Guébec.

\subsection{La propriété de l'eau souterraine et l'État}

L'analyse visant à déterminer si l'eau souterraine est la propriété de l'État impose une incursion hors des frontières du droit provincial. L'eau souterraine est un élément intégré au cycle hydrologique, cycle qui ne reconnaît pas les frontières. Tout comme le droit constitutionnel, le droit international peut être à l'origine de normes juridiques ayant un impact sur le statut juridique de la ressource en droit québécois. Pour étudier l'application de la propriété publique à l'eau souterraine sous chacun de ses aspects, un survol du droit international semble pertinent avant de procéder à une analyse plus précise du droit interne, et notamment du droit constitutionnel, afin d'établir si l'État est propriétaire de la ressource.

d'un terrain a-t-il un droit au support de l'eau sous-jacente, et ce propriétaire a-t-il un droit d'action en responsabilité civile pour les dommages résultant du retrait de cette eau contre l'entrepreneur qui a effectué le pompage? La Cour répond affirmativement à cette question.

109. Ce constat peut être renforcé par le principe du numerus clausus. Selon ce principe, les formes juridiques par lesquelles s'exprime le droit de propriété sont à la fois standardisées et limitées à un nombre maximal. Le numerus clausus a fait l'objet de peu d'attention au Québec, mais la doctrine française reconnaît son existence bien qu'il existe un débat quant à savoir si le principe est une limite substantive reconnue implicitement par le Code civil français, ou résulte plutôt des formalités strictes liées à la mise en oeuvre des droits de propriété. À ce sujet, voir Thomas Merrill \& Henry Smith, "Optimal Standardization in the Law of Property: The Numerus Clausus Principle», (2000) 110 Yale L. J. 1, et en particulier la note 8 à la page 5, ou les auteurs citent notamment Christian Atias, Émile Chénon et Marcel Planiol. L'existence du principe du numerus clausus au Québec diminuerait la capacité d'adaptation du droit de propriété en tant que vecteur capable d'offrir un cadre juridique de gestion économique de l'eau souterraine. 
Le droit international s'articule principalement autour du principe de souveraineté des États. La souveraineté tend vers un contrôle absolu sur les ressources naturelles et le territoire ${ }^{110}$. À prime abord, l'eau souterraine apparaît soumise au contrôle absolu de l'État souverain ${ }^{111}$. Toutefois, la souveraineté étatique en droit international n'emporte pas la propriété112. Le droit international n'offre pas de norme qui puisse imposer un statut juridique à l'eau en droit interne ${ }^{113}$. Une revue sommaire des instruments de droit international relatifs aux eaux souterraines confirme cette conclusion ${ }^{114}$. En fait, le droit international semble être dans une position similaire à celle du droit québécois pour affronter les problèmes de gestion de l'eau souterraine car la souveraineté repose sur la même prémisse de contrôle absolu que la propriété. Or, le contrôle absolu sur la ressource hydrique que

110. Voir l'article 2 de la Charte des droits et devoirs économiques des États, Résolution 3281 (XXIX) de l'Assemblée générale de l'ONU, Doc. N.-U., A/9631 à la p. 53.

111. En fait, la souveraineté territoriale absolue sur les ressources hydriques n'est pas reconnue par le droit international. Les ressources hydriques font plutôt l'objet d'une souveraineté territoriale limitée de la part des États : voir Stephen McCaffrey, The Law of International Watercourses, 2 ième éd., Oxford, Oxford University Press, 2007 aux pp. 111-170.

112. Malcolm Shaw, Title to Territory, Burlington, Ashgate Dartmouth Publishing, 2005 aux pp. 15-16; Les choses communes, supra note 51 à la p. 62.

113. Sylvie Paquerot, Eau douce: la nécessaire refondation du droit international, Québec, Presses de l’Université du Québec, 2005 aux pp. 913. Le droit international économique ne semble pas offrir un statut spécifique à l'eau. Selon Jérôme Dupont-Rachiele, Daniel Prévost \& Sébastien Raymond, "L'eau : un droit pour tous, un bien pour certains", (2004) 17 R.Q.D.I. 39 aux pp. 42-57, l'eau à l'état naturel n'est pas un bien mais l'eau embouteillée l'est, et la tendance est à la marchandisation de l'eau; du débat relatif à ces questions, il est possible de retenir que l'eau souterraine à l'état naturel n'est pas un bien.

114. Voir Stefano Burchi \& Kerstin Melchem, Groundwater in international law: compilation of treaties and other legal instruments, Rome, FAO \& UNESCO, 2005 (ci-après "Groundwater in international law»); Stephen Foster \& Daniel Loucks, dir., Non-renewable groundwater resources; a guidebook on socially-sustainable management for water-policy makers, Paris, UNESCO, 2006 (ci-après "Non-renewable groundwater resources") aux pp. 53-56. 
(2008) 38 R.D.U.S.

La gestion économique

de l'eau souterraine

par le droit de propriété au Québec

la souveraineté offre en théorie est érodé par la réalité concrète d'une dynamique de partage ${ }^{115}$.

Abstraction faite du droit international, la réflexion au sujet de l'appropriation de l'eau souterraine par l'État remonte inévitablement aux sources constitutionnelles du droit116. La Constitution canadienne accorde la propriété des ressources naturelles aux Couronnes provincialesi17. Par conséquent, certaines provinces canadiennes légifèrent pour s'attribuer explicitement la propriété des eaux souterraines ${ }^{118}$. Le législateur québécois peut accorder la propriété de la ressource à l'État de façon expresse mais choisit de ne pas se prévaloir de cette possibilitél19. De plus, certaines dispositions laissent sous-

115. Jochen Sohnle, Le droit international des ressources en eau douce: solidarité contre souveraineté, Paris, Documentation française, 2002 aux pp. 243-270. Les articles 3 à 7 du récent projet d'articles sur le droit des aquifères transfrontières présenté par la Commission du droit international illustrent parfaitement les limites étroites de la souveraineté national à l'égard des eaux souterraines: voir Commission du droit international, Cinquième rapport sur les ressources naturelles partagées : eaux souterraines et transfrontières, Doc. N.U., A/CN.4/591, 60ième session, Genève, 5 mai- 6 juin et 7 juillet- 8 août 2008 .

116. À ce sujet, voir Dale Gibson, "The Constitutional Context of Canadian Water Planning", (1969) 7 Alta. L. Rev. 71 aux pp. 72-73 et Dominique Alhérithière, La gestion des eaux en droit constitutionnel canadien, Québec, Éditeur officiel du Québec, 1976 aux pp. 27-36.

117. Pour le Québec, la propriété provinciale découle généralement des articles 109 et 117 de la Loi constitutionnelle de 1867, R.-U., 30 \& 31 Victoria, c.3 (ci-après "Constitution"), reproduite dans L.R.C. 1985, app. II, n 5 aux pp. 33-34.

118. Voir notamment les articles 1 et 2 de la Loi sur les droits d'utilisation de l'eau, C.P.L.M. c. W80, pour le Manitoba; les articles 1 et 3 al.2 du Water Act, R.S.A., 2000, c. W-3, pour l'Alberta; les articles 2 al. 1 §b) et y), 6 et 9 du Water Resources Act, S.N.L., 2002, c. W-4.01, pour Terre-Neuve. Notons que cet exercice législatif découle nécessairement du pouvoir de l'article 92 (13) Constitution. Selon les mots de François Chevrette, "Dominium et Imperium: l'État propriétaire et l'État puissance publique en droit constitutionnel canadien", dans Benoit Moore, dir., Mélanges Jean Pineau, Montréal, Éditions Thémis, 2003, 665 à la p. 672, la Constitution a mis de l'imperium dans le dominium [Moore].

119. L'article 3 LM énonce que les substances minérales situées sous la couche arable appartiennent au domaine de l'État. Or, la définition de substances minérales de l'article 1 LM exclut l'eau. Cependant, à partir 
entendre que les droits de l'État québécois dans le domaine hydrique sont plus limités que dans le domaine terrestre ${ }^{120}$. Ainsi, il existe une ouverture à la reconnaissance d'un autre statut juridique que celui de bien approprié par l'État pour l'eau souterraine si le droit positif est à cet effet.

Actuellement, le gouvernement québécois semble vouloir placer l'eau souterraine dans un nouveau type de patrimoine, un patrimoine collectif commun à tous les Québécois ${ }^{121}$. Ce nouveau statut juridique impliquerait que l'eau souterraine ne peut être appropriée, sauf dans les conditions définies par la loi, dont le $\mathrm{CCQ}^{122}$. La doctrine québécoise ne semble pas avoir exploré le sujet mais la doctrine française permet d'identifier les effets éventuels de la reconnaissance de l'eau comme patrimoine commun :

De même, la reconnaissance récemment accordée à l'eau en tant que patrimoine commun ne signifie pas que l'État s'approprie l'eau comme étant son patrimoine, mais vise à

du moment où l'eau contient plus de $4 \%$ en poids de solides dissous, c'est-à-dire qu'elle est définie comme saumure, elle appartient à l'État. Notons que la définition de la couche arable se trouve à l'article $1 \S 16^{\circ}$ LPTAA. Cette couche fait rarement plus de $1 \mathrm{~m}$ d'épaisseur selon DenysClaude Lamontagne \& Jean Brisset des Nos, Le droit minier, 2ième éd., Montréal, Éditions Thémis, 2005 aux pp. 5-6.

120. Le mandat qu'exerce le ministre des Ressources naturelles et de la Faune par rapport au domaine terrestre de l'État lui permet d'exercer les droits et pouvoirs inhérents au droit de propriété en lieu et place de la Couronne provinciale : article 2 de la Loi sur les terres du domaine de l'état, L.R.Q., c. T-8.1. Par comparaison, le MDDEP, qui gère le domaine hydrique de l'État, ne dispose que de l'autorité nécessaire pour ce faire, et non de pouvoirs inhérents à la propriété : article 13 de la Loi sur le ministère du Développement durable, de l'Environnement et des Parcs, L.R.Q., c. M-15.2.1. La déduction issue d'une telle comparaison repose sur la prémisse que l'eau souterraine fait partie du domaine hydrique et non du domaine terrestre de l'État. La doctrine ne semble avoir envisagé le domaine hydrique que par rapport aux eaux de surface : Le domaine public foncier, supra note 76 aux pp. 245-327.

121. Voir la Politique nationale, supra note 62 aux pp. 15, 17, 83, 87 et 92. Le projet de reconnaissance juridique des eaux comme patrimoine commun de la nation québécoise est précisé par le Projet de loi no92, supra note 9.

122. Projet de loi $n^{\circ}$ 92, supra note 9, art. 1. 
renforcer sa légitimité à intervenir en qualité de bras séculier de la nation dans l'intérêt de la collectivité qui est susceptible de s'opposer aux intérêts particuliers. Autrement dit, la qualification de patrimoine commun induit une préférence pour l'intérêt collectif. [...]Enfin, le fait que l'intérêt général soit reconnu comme supérieur à l'intérêt individuel entraîne l'apparition d'un devoir de conservation. 123

Ainsi, l'appréhension de la ressource comme un tout soumis à la gestion de l'État peut atténuer la dynamique conflictuelle liée à l'appropriation de la ressource ${ }^{124}$. Par ailleurs, si l'eau constitue un patrimoine commun, l'État en est le gestionnaire125. Les implications d'une telle charge ne sont pas claires. Cependant, le Projet de loi no92 en précise certains éléments : le principe de protection de l'eau pourrait notamment amener le Procureur général, au nom des intérêts de la nation à l'égard des ressources hydriques, à intenter une action en réparation contre l'auteur de dommages causés à ces ressources ${ }^{126}$.

D'autre part, l'élaboration d'un cadre de gestion de l'eau par le biais de la notion de patrimoine commun peut être poursuivie

123. Patrimoine commun, supra note 62 aux pp. 388-389. Pour une étude poussée des questions que soulève l'application de la notion de patrimoine commun par rapport à la propriété, voir Isabelle Savarit, "Le patrimoine commun de la nation, déclaration de principe ou notion juridique à part entière?», (1998) 14 Rev. fr. dr. admin. 305 (ci-après "Patrimoine commun de la nation"). Les conséquences peuvent être importantes en matière d'urbanisme et d'expropriation car la notion de patrimoine commun autorise l'Administration française à apporter des restrictions importantes au droit de propriété du sol qui peuvent aller jusqu'à l'interdiction de construire : Lamy eau, supra note 77, II105-9.

124. Voir L'étrange pérennité, supra note 70 à la p. 168.

125. Voir la Politique nationale, supra note 62 à la p. 83. Selon le préambule du Projet de loi no92, supra note 9, l'État est gardien des intérêts de la nation à l'égard des ressources en eau souterraines qui constituent le patrimoine commun de la nation, et il se doit d'être investi des pouvoirs nécessaires pour en assurer la protection et la gestion.

126. Projet de loi no92, supra note 9, art. 7-9. 
grâce à un rapprochement avec la fiducie ${ }^{127}$. À cet égard, la fiducie offre plusieurs pistes de réflexion qui peuvent avoir une influence directe sur les conséquences juridiques de la caractérisation de l'eau comme patrimoine commun. Premièrement, il semble que la fiducie en droit québécois corresponde à l'institution de la «fiducie expresse" en common law ${ }^{128}$. Or, la common law considère que, dans certains cas, la fiducie expresse peut être définie comme une "fiducie politique" qui n'oblige l'État que moralement et n'est pas justiciable ${ }^{129}$. Toutefois, il semble peu probable que cette conception de la fiducie puisse influencer l'élaboration de la notion de patrimoine commun à l'égard de l'eau puisque le Projet de loi no92 envisage d'emblée la mise en œuvre d'une action en réparation pour protéger le patrimoine hydrique. Deuxièmement, le droit des autochtones a abordé la question de l'obligation fiduciaire de l'État canadien par rapport aux terres ancestrales. Cette charge impose des obligations justiciables à l'État ${ }^{130}$. Cependant, compte tenu des fortes particularités du droit des autochtones, il n'est pas certain que les développements en cette matière à l'égard de la fiducie exercent une influence déterminante à l'égard de l'interprétation des éventuelles obligations fiduciaires de l'État québécois par rapport au patrimoine commun de l'eau ${ }^{131}$.

127. La fiducie n'a pas fait l'objet d'une étude doctrinale au Québec en matière de gestion de l'eau. Toutefois, selon Patrimoine commun de la nation, supra note 123 à la p. 313, la notion de patrimoine commun débouche sur la fiducie, ce qui peut renforcer l'idée que l'État n'est pas propriétaire de la ressource et limiter l'appropriation privée de la ressource : voir l'art. 1261 C.c. $\mathrm{B}$.

128. Madeleine Cantin Cumyn, "L'acte constitutif d'une fiducie» dans Benoît Moore, supra note 118, 649 à la p. 651 .

129. André Lajoie, "Avec des amis comme ceux là... Une double perspective sur les rapports fiduciaires" dans Commission du droit du Canada, Les rapports fiduciaires: une question de confiance, Montréal, Éditions Thémis, 2002, 83 aux pp. 90-91.

130. Voir en général Kent McNeil, «Fiduciary Obligations and Arboriginal People», 807 dans Mark Gillen \& Faye Woodman, dir., The Law of Trusts : A Contextual Approach, Toronto, Emond Montgomery Publications, 2000, 807 aux pp. 813-826.

131. Cependant, dans Guérin c. R., [1984] 2 R.C.S. 335 aux pp. 384-388, la Cour suprême semble suggérer que les principes développés à l'égard de la charge fiduciaire de la Couronne en matière de droit des autochtones 
(2008) 38 R.D.U.S.

La gestion économique

de l'eau souterraine

par le droit de propriété au Québec

Troisièmement, il existe un concept de "fiducie publique" en droit américain qui a été utilisé notamment pour limiter les droits découlant de l'appropriation de l'eau ${ }^{132}$. La fiducie publique peut aussi obliger l'État à gérer les ressources publiques afin qu'elles restent disponibles pour le public. Le défaut de respecter cette obligation fiduciaire est justiciable. Cependant, il semble que cette doctrine ne soit pas encore reçue au Canada133. Néanmoins, des développements jurisprudentiels récents indiquent que la reconnaissance de cette doctrine est désormais possible, bien que ses implications juridiques ne soient pas encore précisément identifiées ${ }^{134}$. Les nombreuses références à l'«intérêt public" dans le Projet de loi no92 renforcent l'impression que l'élaboration des principes relatifs à la notion de patrimoine commun pourrait être liée à la fiducie publique ${ }^{135}$. Finalement, il est aussi possible d'esquisser les éventuels contours des obligations fiduciaires de l'État à l'égard du patrimoine commun hydrique par référence aux

soient en relation avec le droit de la fiducie au-delà des limites du droit des autochtones.

132. Scott Kidd, "Keeping Public Ressources in Public Hands: Advancing the Public Trust Doctrine in Canada", (2006) 16 J. Envtl. L. \& Prac. 187 à la p. 194.

133. Donovan Waters, Waters' Law of Trusts in Canada, 3ième éd., Toronto, Thomson Carswell, 2005 aux pp. 567-568.

134. Selon la Cour suprême dans Colombie Britannique c. Canadian Forest Products, [2004] 2 R.C.S. 74, para. 81, "It seems to me there is no legal barrier to the Crown suing for compensation as well as injunctive relief in a proper case on account of public nuisance, or negligence causing environmental damage to public lands, and perhaps other torts such as trespass, but there are clearly important and novel policy questions raised by such actions. These include the Crown's potential liability for inactivity in the face of threats to the environment, the existence or nonexistence of enforceable fiduciary duties owed to the public by the Crown in that regard, the limits to the role and function and remedies available to governments taking action on account of activity harmful to public enjoyment of public resources, and the spectre of imposing on private interests an indeterminate liability for an indeterminate amount of money for ecological or environmental damage." À cet égard, voir aussi Jerry DeMarco, "The Supreme Court of Canada's Recognition of Fundamental Environmental Value: What Could be Next in Canadian Environmental Law?», (2007) 17 J. Env. L. \& Practice 159 aux pp. 195203.

135. Par exemple, voir Projet de loi no92, supra note 9, art. 17-31.81. 
règles qui gouvernent les fiducies en droit civil136. D'abord, l'État maîtriserait et administrerait pleinement les eaux en fiducie ${ }^{137}$. Il devrait conserver et faire fructifier le patrimoine commun, conformément au meilleur intérêt de la collectivité québécoise ${ }^{138}$. L'État ne pourrait exercer ses pouvoirs fiduciaires dans un autre intérêt que celui de la collectivité139. Bien qu'il puisse déterminer les parts respectives des divers bénéficiaires parmi la collectivité québécoise, l'État serait tenu d'agir avec impartialité140. Ce faisant, le patrimoine commun pourrait être grevé de droits réels mais ne pourrait faire l'objet d'une disposition à titre gratuit ${ }^{141}$. Finalement, il serait possible d'agir contre l'État afin de le contraindre à exécuter ses obligations de fiduciaire, et à faire un acte nécessaire ou à ne pas faire un acte dommageable pour le patrimoine commun ${ }^{142}$.

En somme, le droit statutaire montre que l'État québécois évite expressément de se déclarer propriétaire de l'eau souterraine. Ses droits à l'égard du domaine hydrique apparaissent moins étendus que les droits de propriété qu'il exerce par rapport aux terres de la Couronne. Par ailleurs, le gouvernement québécois veut faire de l'État le gardien et gestionnaire en fiducie d'un patrimoine hydrique commun appartenant à la nation québécoise. Bien que la reconnaissance de l'eau comme patrimoine commun puisse éventuellement déboucher sur une gestion économique de la ressource par l'action législative et réglementaire de l'État fiduciaire, ni la propriété publique ni la notion de patrimoine

136. La fiducie de type social semble particulièrement pertinente car elle réfère à l'intérêt général qui se retrouve aussi à l'art. 913 C.c.Q. (voir cidessous 2.4) de même qu'à l'art. 3 du Projet de loi no92, supra note 9.

137. Art. 1278 C.c.Q.

138. Art. 1306,1309 C.c.Q.

139. Art. 1310,1311 C.c.Q.

140. Art. 1282,1283 et 1317 C.c.Q. Notons que l'impartialité est exigible par les bénéficiaires successifs, ce qui est particulièrement pertinent dans un contexte de développement durable où les générations futures doivent recevoir ou disposer d'autant que les générations présentes.

141. Art. 1307,1315 C.c.Q.

142. Art. 1290 C.c.Q. 
(2008) 38 R.D.U.S.

La gestion économique

de l'eau souterraine

par le droit de propriété au Québec

commun ne permettent en elles-mêmes la gestion économique de l'eau souterraine.

\subsection{L'eau souterraine : res communis}

Une étude détaillée montre que la propriété privée de même que ses modalités et démembrements ne peuvent fournir un cadre juridique cohérent de gestion à l'égard des eaux souterraines. De plus, le législateur québécois évite de donner à l'eau souterraine le statut de bien domanial. Ce contexte juridique permet de conclure, suivant les arguments avancés dans la présente souspartie, que l'eau souterraine est une res communis en vertu d'une disposition expresse. En effet, la loi ne mentionne jamais que l'eau dans son état naturel, en surface ou dans le sol, est susceptible d'être appréhendée par la propriété143. Par contre, le CCQ mentionne expressément que l'eau est d'usage commun à tous :

913. Certaines choses ne sont pas susceptibles d'appropriation; leur usage, commun à tous, est régi par des lois d'intérêt général et, à certains égards, par le présent code.

L'air et l'eau qui ne sont pas destinés à l'utilité publique sont toutefois susceptibles d'appropriation s'ils sont recueillis et mis en récipient.

A priori, l'eau est donc une chose commune hors de la portée du droit de propriété ${ }^{144}$. La référence aux lois d'intérêt général contenue à l'article $913 \mathrm{CCQ}$ permet d'établir que l'eau souterraine est effectivement visée par cette disposition ${ }^{145}$. En

143. L'eau, chose commune, supra note 61 à la p. 410.

144. Morin c. Morin, [1998] R.J.Q. 23 (C.A.) (ci-après "Morin c. Morin") à la p. 28.

145. Pierre Issalys \& Denis Lemieux, L'action gouvernementale : précis de droit

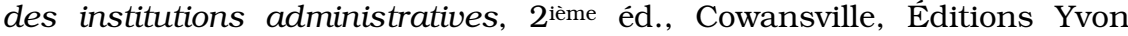
Blais, 2002 (ci-après "L'action gouvernementale») aux pp. 67-70, fournissent une définition des termes "intérêt général" qui aide à comprendre l'interaction du régime de protection et de gestion de l'environnement avec l'intérêt privé dont la propriété permet l'épanouissement : "Il existe une présomption absolue à l'effet que toute loi est adoptée pour promouvoir l'intérêt général [...] Ce concept d'intérêt 
effet, la LQE, qui est une loi d'intérêt général, définit l'eau comme étant l'eau de surface et l'eau souterraine où qu'elles se trouvent146. Ainsi, l'eau souterraine est une res communis. Cependant, le deuxième alinéa de l'article 913 CCQ indique que le statut de l'eau souterraine comme res communis n'est pas constant. L'eau souterraine peut être un bien approprié si elle a deux caractéristiques cumulatives: 1) ne pas être d'utilité publique; 2) être recueillie et mise en récipient.

Premièrement, l'eau souterraine doit être destinée à l'utilité publique pour être res communis. La notion d'utilité publique est vague. Elle accorde un large pouvoir interprétatif aux tribunaux qui sont appelés à la définir. En contrepartie, elle confère à la notion de res communis une souplesse qui permet de faire face à la réalité du commerce de l'eau.

La notion d'utilité publique n'est pas étudiée en détail par la jurisprudence dans le cadre de l'article 913 CCQ $^{147}$. Toutefois, le volume d'eau et le nombre de personnes qui utilisent l'eau dont on tente de déterminer le caractère d'utilité publique semblent être des facteurs pouvant influencer la décision des tribunaux ${ }^{148}$. Pourtant, ces deux facteurs sont incapables de rendre pleinement

général s'oppose à celui d'intérêt privé ou particulier. [...] En principe, l'intérêt général est d'abord et avant tout l'ensemble des intérêts communs de la collectivité, même s'il pourra souvent coïncider avec l'intérêt spécial d'un citoyen. [...] L'intérêt général pourra s'opposer à l'intérêt individuel ou à celui d'une collectivité donnée. [...] L'intérêt général est, en quelque sorte, fragmenté par les lois en vigueur et chacune d'elle en véhicule une partie sous forme d'intérêt public particulier. [...] Aussi, un but d'intérêt public identifié dans une loi pourra s'opposer à un autre but, lui aussi d'intérêt général, mais non reconnu par la loi.» (Citations et références omises).

146. Art. $1 \S 1^{\circ}$ LQE.

147. L'affaire Gauthier c. Côté, supra note 68, semble la seule qui traite du sujet sous l'article 913 C.c. $Q$

148. Gauthier c. Côté, supra note 68 à la p. 7; Rouleau c. Station MontTremblant, J.E. 2003-555 (C.S.) aux pp. 8-11. Aussi à l'effet que le nombre de personnes bénéficiaires est pertinent pour déterminer le caractère d'utilité publique, mais dans un contexte étranger au domaine hydrique, voir École de technologie supérieure c. Société d'ingénierie C.I.M.A., [1997] R.J.Q. 2852 (C.S.) aux pp. 2863-2866. 
(2008) 38 R.D.U.S.

La gestion économique

de l'eau souterraine

par le droit de propriété au Québec

compte de l'utilité publique d'une source d'approvisionnement parce qu'ils font abstraction des fonctions essentielles que la ressource remplit auprès de ses usagers ${ }^{149}$. L'utilité publique doit aussi être déterminée par rapport à la fonction d'un objet, c'est-àdire sa finalité. D'ailleurs, les développements relatifs à la notion d'utilité publique dans le cadre d'autres dispositions du CCQ permettent de qualifier l'objet par rapport à sa fonction ${ }^{150}$. Par exemple, la théorie de l'accessoire entraîne la reconnaissance de l'utilité publique d'un objet même si le public n'y a pas un accès général ${ }^{151}$.

D'autre part, quelques dispositions accordent des droits d'utilisation spécifiques par rapport à certaines eaux. Ainsi, toute personne a le droit de circuler sur les cours d'eau et les lacs à condition de respecter certaines modalités ${ }^{152}$. La jurisprudence reconnaît clairement le lien entre l'article 913 CCQ et le droit de

149. Si le volume d'eau et le nombre de bénéficiaires sont les deux seuls critères permettant de déterminer l'utilité publique de la ressource, il est possible de se poser la question suivante : la source d'eau souterraine qui approvisionne les 30 utilisateurs du réseau municipal Pointe-Parent dans la municipalité de Natashquan est-elle d'utilité publique, compte tenu notamment du nombre de personnes desservies par rapport à la population totale de la municipalité (374 personnes)? Que la question se pose révèle d'emblée l'insuffisance de ces deux critères de qualification.

150. Voir par exemple les art. 916 et 952 C.c.Q. L'affaire Commission Scolaire Saint-Jérome c. Alco-Tek Électrique inc., [1997] R.D.I. 234 (C.S.), définit l'utilité publique sous l'art. 916 C.c.Q. Le droit de l'expropriation permet de saisir la relation entre l'utilité publique mentionnée et la fonction ou la finalité d'un objet : l'expropriation est justifiée par l'utilité publique selon l'art. 952 C.c.Q. et l'autorité qui exproprie doit indiquer la finalité de l'expropriation selon l'article 40 al.2 de la Loi sur l'expropriation, L.R.Q. c. E-24. Par ailleurs, lorsqu'une loi prévoit expressément un motif d'expropriation, il faut présumer que l'objet qui peut être exproprié est d'utilité publique. L'eau qui peut servir de source d'approvisionnement au sens des articles 41 et 42 LQE est donc d'utilité publique car elle peut être visée dans le cadre d'une expropriation par une municipalité ou un exploitant d'aqueduc privé.

151. Bâtiments Kalad'Art inc., c. Construction D.R.M. inc., [2000] R.J.Q. 72 (C.A.) aux pp. 75-77; Biens et propriété, supra note 34 aux pp. 145-147.

152. Art. 920 C.c.Q. 
circulation sur les eaux de surface ${ }^{153}$. Un tel droit confère aux eaux de surface une utilité publique, ce qui permet de mieux définir le domaine d'application de l'article 913 CCQ par rapport aux eaux : notamment, les eaux sur lesquelles l'exercice du droit de circulation est possible sont des res communes insusceptibles d'appropriation selon le droit commun de la propriété154.

Le deuxième alinéa de l'article 913 CCQ introduit une nuance qui aide à clarifier la portée de cette disposition dans un tel contexte : il n'est pas nécessaire que l'utilité publique de la ressource soit immédiate; plutôt, il est suffisant que l'eau soit destinée à l'utilité publique. À ce point, la réalité du cycle hydrologique doit être prise en compte. À l'étiage, le débit d'un cours d'eau ne peut provenir, en l'absence de toute pluviométrie, que de l'écoulement des nappes souterraines. De même, tout prélèvement dans une nappe provoque une diminution du débit des sources et des cours d'eau alimenté par la nappe ${ }^{155}$. À terme, l'écoulement de l'eau souterraine permet l'exercice du droit de circulation. Compte tenu de l'unité du cycle hydrologique, toute eau à l'état naturel, où qu'elle se trouve, peut être considérée $a$ priori comme res communis. Dans une certaine mesure, il est alors possible d'envisager une reconnaissance légale $\mathrm{du}$ cycle hydrologique liée à la notion de res communis.

Cette amorce de reconnaissance est renforcée par la servitude d'écoulement, selon laquelle les terrains en aval hydraulique sont assujettis à recevoir toutes les eaux qui

153. Morin c. Morin, supra note 144 aux pp. 28-29; Association des résidents du lac Mercier inc. c. Paradis, [1996] R.J.Q. 2370 (C.S.) à la p. 2378.

154. Dans Charlotte Lemieux, "La protection de l'eau en vertu de l'article 982 C.c.Q. : problèmes d'interprétation", (1992) 23 R.D.U.S. 191 à la p. 198, le lien entre la définition d'utilité publique de l'art. 913 C.c.Q. et le droit d'utilisation accordé par l'art. 920 C.c.Q. est identifié. Toutefois, Charlotte Lemieux établit une distinction entre l'usage public (ex. : art. 920 C.c.Q.) et l'utilité publique. Une telle distinction paraît artificielle et n'est pas retenue par la Cour d'appel dans l'affaire Morin c. Morin, supra note 144 aux pp. 28-29.

155. Lamy eau, supra note 77, II105-4. 
(2008) 38 R.D.U.S.

La gestion économique

de l'eau souterraine

par le droit de propriété au Québec

découlent naturellement des terrains en amont hydraulique ${ }^{156}$. La servitude d'écoulement contraint à la fois le propriétaire du terrain en aval hydraulique, qui ne peut empêcher cet écoulement par la construction d'un ouvrage, et le propriétaire du terrain en amont, qui ne peut généralement pas aggraver la situation du terrain en aval157. Finalement, à l'égard des eaux de surface, la

156. Art. 979 C.c.Q. Notons cependant que la servitude d'écoulement est profondément altérée par le régime que met en place la Loi sur le régime des eaux, L.R.Q., c. R-13 (ci-après "LRE»). Cette loi crée une "servitude légale d'inondation": Louise Ouellet, "La portée juridique de certaines dispositions de la Loi sur le régime des eaux", 141 à la p. 145, dans Service de formation permanente du Barreau du Québec, Développements récents en droit de l'environnement (1999), Cowansville, Éditions Yvon Blais, 1999. Dans ce cas, l'intérêt général repose sur le développement économique et requiert que le cycle hydrologique subisse des modifications: Lorne Giroux, Marie Duchaîne, Gilbert Noreau \& Johanne Vézina, "Le régime juridique applicable aux ouvrages de retenue des eaux au Québec", (1997) 38 C. de D. 3 aux pp. 6-7.

157. Art. 979 C.c.Q. Le propriétaire du terrain d'amont peut toutefois se prévaloir d'exceptions lui permettant de modifier l'écoulement de l'eau. Par ailleurs, l'interprétation de l'art. 979 C.c.Q. limitant les contraintes applicables au propriétaire du terrain en amont hydraulique doit être écartée. Contrairement au courant jurisprudentiel auquel appartiennent les affaires Brousseau c. Martel, [1990] R.D.I. 620 (C.S.) à la p. 623 et Orychiwsky c. Murphy, [2005] R.DI. 109 (C.S.) aux pp. 113-114, il est nécessaire d'accorder tout son sens à l'art. 979 C.c.Q. Selon ces décisions, un ouvrage réalisé sur le terrain d'amont qui empêche les eaux de couler naturellement vers le terrain en aval ne constitue pas une aggravation de sa situation. Pourtant, il est difficile de saisir pourquoi l'installation d'un ouvrage de captage d'eau souterraine par le propriétaire du terrain supérieur, installation qui empêche l'écoulement naturel vers le terrain inférieur où le voisin ne peut plus procéder au pompage d'eau souterraine à ses propres fins, ne constitue pas une aggravation. Cependant, ces jugements doivent être compris dans le contexte historique et hydrographique québécois. Compte tenu de l'abondance de l'eau dans nos régions, la jurisprudence traite principalement de litiges causés par un surplus d'eau. De plus, les connaissances relatives aux autres eaux que celles des cours d'eau et des lacs sont relativement récentes et les problématiques qui y sont liées n'ont pas encore été parfaitement intégrées par le droit civil traditionnel. C'est pourquoi l'art. 981 C.c.Q., qui accorde un droit d'usage restreint sur les eaux de surface, est traditionnellement considéré comme le pendant naturel de l'art. 979 C.c.. . : puisque les eaux principalement appréhendées par le droit civil traditionnel dans le cadre de ces articles 
reconnaissance implicite du cycle hydrologique est complétée par l'interdiction de toute modification importante des débits ou des volumes hydriques ${ }^{158}$.

Deuxièmement, les eaux souterraines ne peuvent être appropriées avant d'être recueillies et mises en récipient ${ }^{159}$. D'une part, l'eau d'une source dont il est possible d'user et de disposer n'est un bien appropriable sujet au droit de propriété que si cette eau est recueillie et mise en récipient par le propriétaire du terrain ${ }^{160}$. L'eau souterraine à l'état naturel est toujours une res communis. La ressource cesse d'être une res communis uniquement lorsqu'elle est contenue dans un récipient. Tant qu'elle n'est pas confinée à un récipient, la propriété de l'eau souterraine échappe au propriétaire immobilier ${ }^{161}$. D'autre part, le texte du deuxième alinéa de l'article 913 CCQ implique indéniablement que le recueillement en récipient ne peut priver une eau destinée à l'utilité publique du statut de res communis. La distinction entre res communis et res nullius acquiert ainsi une signification par rapport aux eaux souterraines : la véritable eau res communis - c'est-à-dire celle dont l'appropriation est empêchée par l'effet du droit et non pas parce que personne n'y a encore

sont les eaux de surface, il n'a pas encore été considéré comme nécessaire d'accorder tout son sens à l'art. 979 C.c.Q.

158. Art. 981 C.c.Q. L'affaire Morin c. Morin, supra note 144 aux pp. 28-29, identifie aussi certaines eaux visées à l'art. 981 C.c.Q. comme d'utilité publique au sens de l'art. 913 C.c. Q. Il faut préciser que les volumes qui peuvent être utilisée par le propriétaire riverain sans modification quantitative importante ne font pas partie des eaux d'utilité publique.

159. Voir Amos (Ville d') c. Loranger, [1999] T.A.Q. 1021 à la p. 1029.

160. Ici, les droits d'user et de disposer font référence aux droits accordés par l'art. 980 C.c.Q. Selon les commentaires du ministre de la Justice lors de la réforme du droit civil, la règle énoncée par l'art. 980 C.c.Q. est en accord avec l'art. 913 C.c.Q. : Commentaires du ministre, supra note 38 à la p. 330.

161. Il faut donc conclure que l'art. 980 C.c.Q. n'accorde pas de droit de propriété, ou un démembrement ou une modalité quelconque du droit de propriété à l'égard de la ressource mais seulement un droit d'utilisation sur certaines eaux. Cette conclusion est conforme à la doctrine : L'eau, chose commune, supra note 61 à la p. 409; Bilan de la réforme, supra note 42 à la p. 328-330. 
(2008) 38 R.D.U.S.

La gestion économique

de l'eau souterraine

par le droit de propriété au Québec

songé ou n'en a les moyens techniques - est l'eau d'utilité publique.

En somme, la combinaison des deux caractéristiques cumulatives prévues à l'article 913 CCQ permet d'établir que l'eau souterraine à l'état naturel est toujours une chose commune, quelle que soit sa situation particulière. Puisque le statut de res communis implique que la propriété soit inapplicable à la ressource, la propriété ne peut pas directement constituer, sous quelque forme que ce soit, un cadre juridique permettant la gestion économique de l'eau souterraine ${ }^{162}$. Il reste alors à déterminer si la caractérisation de la ressource comme chose commune impose un cadre juridique en matière de gestion de l'eau souterraine.

Cette question commence à peine à faire l'objet d'une attention particulière en droit québécois ${ }^{163}$. La doctrine française tente depuis plus longtemps d'établir que la notion de res communis implique un mode de gestion spécifique ${ }^{164}$. Le trait essentiel de ce mode de gestion reposerait sur l'utilisation sans dégradation qui ne fait pas obstacle à un même usage par autrui. À l'égard de l'eau, un tel mode de gestion semble inapplicable ${ }^{165}$. L'eau qui est bue en fournit un bon exemple : un premier usage alimentaire rend impossible la réutilisation directe et immédiate de la ressource aux fins d'alimentation. L'eau usagée est rejetée dans l'environnement et suit le cheminement du cycle hydrologique afin d'être recyclée et purifiée pour finalement retomber sous forme de pluie en amont du point de captage d'un réseau d'aqueduc avant d'être bue à nouveau. Par ailleurs, si le mode de gestion qu'impose la caractérisation comme res communis vise des usages successifs de même nature aussi éloignés que

162. En particulier, voir la section $1.3 \mathrm{du}$ texte.

163. Voir Madeleine Cantin Cumyn, "Le régime juridique de l'eau, chose commune", dans Catherine Choquette \& Alain Létourneau, Vers une gouvernance de l'eau au Québec, Québec, Éditions Multimondes, 2008 ["Le régime juridique de l'eau, chose commune»].

164. Voir notamment Droit de l'environnement, supra note 44 à la p. 149.

165. Voir supra note 105. 
ceux qui sont identifiés dans cet exemple, le critère d'utilisation ne faisant pas obstacle à un même usage par autrui perd toute valeur opérationnelle spécifique puisqu'il s'applique aussi bien à l'eau embouteillée, parfaitement appropriée, qu'à l'eau chose commune. En somme, le mode de gestion des res communes proposé par la doctrine française est inadéquat car il ne reconnaît pas le caractère consomptible inhérent de certaines choses communes.

À l'égard des implications de la qualification comme res communis sur la gestion de l'eau souterraine, une autre piste semble plus prometteuse. Selon l'article 3 du Projet de loi no92, la protection, la restauration, la mise en valeur et la gestion des ressources en eau seraient d'intérêt général. Compte tenu de l'utilisation de la même notion d'intérêt général au premier alinéa de l'article 913 CCQ, il est possible d'imaginer deux conséquences par rapport à la qualification de la ressource comme chose commune suite à la sanction du Projet de loi no92: le droit statutaire ayant trait à la gestion des ressources hydriques devrait respecter les principes énoncés à l'article 3 du Projet de loi no92; en l'absence de norme législative ou réglementaire applicable à l'un des aspects spécifiques de la gestion de l'eau, toute opération visant la ressource à l'état naturel devrait respecter les principes énoncés à l'article 3 du Projet de loi no92.

Quoi qu'il en soit, avant que le Projet de loi no92 ne soit sanctionné, il semble préférable de limiter au droit effectif l'analyse des effets de la caractérisation comme chose commune sur la gestion de l'eau souterraine. L'article 913 CCQ indique d'abord que l'eau souterraine est d'usage commun à tous. Cette seule indication est insuffisante pour permettre une gestion effective de la ressource par le biais de la notion de res communis : elle implique que toute personne peut utiliser la chose commune sans indiquer la qualité que l'eau doit conserver, ni identifier les volumes pour lesquels son usage est accordé ou encore les fins auxquelles elle peut servir. A priori, l'article 913 CCQ semble offrir 
(2008) 38 R.D.U.S.

Lagestion économique

de l'eau souterraine

par le droit de propriété au Québec

libre accès à l'eau souterraine. Or, une telle situation mène à la spoliation de la ressource ${ }^{166}$.

Cependant, l'article 913 ajoute que l'eau est régie par des lois d'intérêt général et, à certains égards, par le CCQ ${ }^{167}$. Puisque le droit statutaire restreint l'usage de la ressource et autorise l'accès à des volumes déterminés, l'eau souterraine n'est effectivement pas libre d'accès ${ }^{168}$. De plus, le droit commun de la propriété en régit aussi l'utilisation. La propriété immobilière accorde au propriétaire un droit d'utilisation par rapport à n'importe quelles eaux touchant un immeuble169. Ce droit n'est pas compris dans l'usus lié à la propriété du terrain; il n'est pas inclus dans le droit de propriété mais est plutôt exorbitant de ce dernier ${ }^{170}$. Sa position dans le CCQ indique qu'il s'agit d'un droit

166. À ce sujet, voir supra note 103, de même que Edella Schlager, "CommonPool Resource Theory" dans Robert Durant, Daniel Fiorino \& Rosemary O'Leary, dir., Environmental governance reconsidered : challenges, choices and opportunities, Cambridge, MIT Press, 2004, 145.

167. Il s'agit d'une solution qui rejoint celle que propose le droit français. Selon Jean-Yves Cherot, "Droit et environnement: introduction au séminaire" dans Jean-Yves Cherot \& al., Droit et environnement : propos pluridisciplinaire sur un droit en construction, Aix-en-Provence, Presses universitaires d'Aix-Marseille, 1995, 7 à la p. 10 : "[1]e régime d'utilisation de la chose commune ne passe donc pas par des arrangements entre les usagers. La réglementation de l'usage de la chose doit être établie par l'autorité de police."

168. Pour une étude du régime statutaire de gestion de l'eau souterraine, voir Hugo Tremblay, Étude du cadre juridique de gestion quantitative des eaux souterraines au Québec, Essai de maîtrise, Québec, Université Laval, 2007 aux pp. 43-78 [Gestion quantitative des eaux souterraines].

169. Art. 980 al.1 C.c.Q.; voir aussi supra note 161. Par ailleurs, l'interprétation de l'ensemble des art. 979-981 C.c.Q. implique que la notion de "source" utilisée à l'art. 980 C.c.Q. n'est plus considérée comme liée à un objet matériel concret tels une ouverture dans la roche ou un jaillissement d'eau. La notion de source réfère plutôt à l'accès à toute eau qui touche à la propriété immobilière, ce qui semble plus conforme à la réalité hydrologique et à l'unicité de la ressource. La référence à la notion de source dans l'énumération de l'art. 982 C.c.Q. doit être comprise comme une mention générique englobant les exemples suivants, ce qui correspond à l'ordre de l'énumération.

170. Cette interprétation de l'ensemble des art. 979-981 C.c.Q. affranchie du droit de propriété sur les eaux est cohérente avec les développements 
réel, ou in rem, attaché au sol et non à la personne. Un propriétaire l'acquiert et le perd en même temps qu'il acquiert et vend un terrain. Ce droit permet notamment de modifier l'écoulement naturel des eaux ${ }^{171}$. Cependant, les volumes desquels il permet l'usage restent a priori indéterminés. Les règles de bon voisinage s'y appliquent et peuvent éventuellement entraîner l'identification précise des quantités auxquelles le propriétaire a droit en cas de litige. Toutefois, en l'absence de voisins, le droit d'utilisation offre effectivement au propriétaire un accès libre à l'eau selon le droit commun de la propriété172. Dans ce contexte, il est possible d'identifier le droit de propriété immobilière comme étant la condition essentielle qui donne naissance au droit d'utilisation de l'eau souterraine ${ }^{173}$.

Par ailleurs, la propriété permet aussi au titulaire de monopoliser l'accès à l'eau et l'usage de la ressource sur sa

présentés ci-dessus et reproduit l'interprétation des art. 993-996 C.c.Q. sur les vues. Les dispositions sur les vues partagent le même contexte de voisinage immobilier que les dispositions relatives aux eaux. Or, les dispositions sur les vues gèrent la circulation de la lumière d'une propriété immobilière à l'autre. Pourtant, personne ne prétend à la propriété de la lumière. De même en est-il de la circulation de l'eau, qui est gérée par les art. 979-981 C.c.Q. sans que la propriété ne s'attache à la ressource.

171. Voir les art. 980 al.2 et 981 C.c.Q., qui constituent des exceptions à l'obligation contenue à l'art. 979 C.c.Q. de laisser l'eau s'écouler naturellement. Notons que le droit d'utilisation de l'eau est restreint par rapport à certaines eaux de surface. En vertu du droit civil, les droits d'utilisation de l'eau souterraine sont donc plus larges que les droits d'utilisation sur les eaux de surface.

172. À l'égard des litiges entre voisins relativement aux eaux souterraines, voir notamment les art. 976 et 982 C.c.Q., de même que Gestion quantitative des eaux souterraines, supra note 168 aux pp. 95-102.

173. Il s'agit de la seule interprétation qui donne un sens à la fois aux mots "régi... par le présent code" de l'art. 913 al.1 C.c.Q. et tout son sens à l'art. 980 al.1 C.c. . Par exemple, si le droit d'usage accordé à tous par l'art. 913 al.1 C.c.Q. subsistait sur la propriété immobilière d'un tiers, l'art. 980 al.1 C.c.Q. serait inutile puisque son sens serait déjà inclus dans le droit d'usage de l'art. 913 C.c.Q. L'art. 980 al.1 C.c.Q. n’a une utilité que s'il accorde au propriétaire immobilier un droit que d'autre n'ont pas, c'est-à-dire le droit d'usage de l'eau touchant son immeuble sur son immeuble. 
propriété immobilière en excluant les tiers, sous réserve des droits publics tels que le droit de navigation ${ }^{174}$. L'existence d'un droit indéterminé d'utilisation de l'eau souterraine institue de facto une classe de personnes ne pouvant utiliser l'eau souterraine faute d'accès à la propriété immobilière ${ }^{175}$. Ainsi, le droit civil induit de façon indirecte une répartition quantitative bivalente de l'eau souterraine entre les propriétaires et les non-propriétaires. Ce mécanisme de gestion est primitif car il ne permet pas la gestion de l'eau souterraine selon des quantités ou qualités spécifiques mais accorde l'équivalent d'un droit d'accès dont la portée est $a$ priori indéfinie. Puisque le cadre juridique offert par le droit civil à l'égard de la chose commune est imprécis aux niveaux quantitatif et qualitatif, et ne découple pas le droit de propriété immobilière du droit d'utilisation de l'eau, ce cadre n'engage pas la gestion de la ressource selon une approche économique.

\section{CONCLUSION}

En théorie, le droit de propriété permet la gestion économique de l'eau et l'apparition de mécanismes de marché s'il offre un cadre juridique applicable à la ressource qui réunit les caractéristiques suivantes: il définit et établit précisément la quantité et la qualité d'eau qui lui est sujette; il rompt les liens

174. Comme le mentionne Le régime juridique de l'eau, chose commune, supra note 163, "Lorsque le sol est la propriété d'un particulier, il ne serait pas possible d'autoriser l'accès de tiers à la source sans entamer de façon décisive la jouissance du propriétaire du fonds. La règle qui privilégie ce dernier n'entraîne pas pour autant comme conséquence nécessaire qu'il ait la propriété de l'eau de la source. L'exclusion des tiers reste compatible avec la qualification de l'eau de la source comme une chose commune: elle admet que, dans ces circonstances particulières, la source sert exclusivement à satisfaire les besoins en eau du propriétaire du fonds." Une autre interprétation accorerait une portée si large à l'art. 913 C.c. Q. que le droit d'usage prévu à cet article provoquerait un affaiblissement critique du droit de propriété immobilière tel qu'exercé traditionnellement.

175. Cette affirmation constitue un énoncé général qui laisse place aux divers droits réels et personnels. Un usufruitier ou même un locataire peuvent avoir accès aux eaux à l'état naturel, mais cet accès reste conditionnel à la volonté initiale du propriétaire qui accepte l'usufruit ou la location. 
juridiques entre les droits de propriété de l'eau et les droits de propriété du sol; il permet le transfert des droits de propriété sur l'eau d'une personne à l'autre contre compensation économique; les droits de propriété sont permanents ou valides sur une période suffisamment longue pour avoir une réelle valeur par rapport à des acheteurs potentiels; les droits de propriété sont protégés par l'Administration et les tribunaux, opposables aux tiers et, le cas échéant, justiciables.

L'analyse du cadre juridique relatif aux diverses formes de la propriété de l'eau souterraine permet de conclure que la propriété ne s'applique pas à la ressource. D’abord, la propriété privée est incapable d'appréhender l'eau souterraine et les tentatives en ce sens induisent des incohérences fragilisant la structure du droit commun de la propriété en tant que régime de gestion des ressources. De plus, même si elle s'y appliquait, la propriété privée lierait la propriété de l'eau souterraine à celle du sol et ne permettrait pas de définir les volumes et la qualité de l'eau sur lesquels le titre porterait. Ensuite, les modalités et démembrements de la propriété privée qui offrent potentiellement des manifestations concrètes de la propriété commune de l'eau souterraine dans le contexte québécois ne constituent pas un cadre de gestion adéquat de la ressource car ils sont imprécis, ne sont pas assez souples pour tenir compte des schémas d'usage actuels, et sont incapables de prendre en considération la réalité hydrologique. Finalement, la propriété publique n'a pas d'impact direct sur la gestion de l'eau souterraine parce que l'État québécois évite spécifiquement de s'approprier la ressource. Au contraire, l'eau souterraine à l'état naturel est une chose commune par l'effet d'une disposition expresse, et l'appropriation de la ressource est impossible.

Ainsi, le droit de propriété, sous quelque forme que ce soit, ne fournit pas directement de cadre juridique permettant une gestion économique de la ressource. Dans la mesure où d'éventuels instruments statutaires de gestion économique sont mis en place, l'action des autorités législatives et réglementaires est libre des contraintes qui auraient pu découler de l'application 
(2008) 38 R.D.U.S.

La gestion économique

de l'eau souterraine

539

par le droit de propriété au Québec

directe de la propriété à la ressource et du chevauchement de deux cadres juridiques visant le même objet.

Néanmoins, le droit commun de la propriété a une influence indirecte sur la gestion de la ressource en tant que chose commune car il en restreint l'usage. Le CCQ prévoit que le cadre général des droits d'utilisation de la ressource est fourni par la servitude d'écoulement : en principe, l'écoulement naturel de l'eau doit rester libre176. Toutefois, la propriété immobilière est liée à un droit d'utilisation par rapport à n'importe quelles eaux touchant l'immeuble177. Ce droit permet de modifier l'écoulement naturel des eaux ${ }^{178}$. Ainsi, le droit civil institue de facto une classe de personnes ne pouvant utiliser l'eau souterraine faute d'accès à la propriété immobilière. Le statut de propriétaire immobilier induit de façon indirecte une répartition quantitative bivalente de la ressource. Ce mécanisme est primitif car il ne permet pas la gestion économique de l'eau souterraine selon des quantités spécifiques mais accorde a priori l'équivalent d'un droit d'accès dont la portée est indéfinie ${ }^{179}$.

176. Art. 979 C.c.Q.

177. Art. 980 al.1 C.c.Q.

178. Toutefois, le droit d'utilisation de l'eau est restreint par rapport à certaines eaux de surface : art. 980 al.2 et 981 C.c. $Q$.

179. Toutefois, les art. 976 et 982 C.c.Q. encadrent l'art. 980 al.1 C.c.Q. et offrent un moyen de dernier recours pour répartir précisément la ressource en cas de conflit. 
